\title{
Early Statins after Intravenous or Endovascular Recanalization Is Beneficial Regardless of Timing, Intensity, and Stroke Mechanism
}

\author{
Han-Gil Jeong, Beom Joon Kim, Mi Hwa Yang, Moon-Ku Han, Hee-Joon Bae \\ Department of Neurology and Cerebrovascular Center, Seoul National University Bundang Hospital, Seongnam, Korea
}

\section{Dear Sir:}

In the modern era of ischemic stroke treatment, stroke physicians have highly effective tools to recanalize occluded major cerebral arteries. After hyperacute treatments, deleterious effects of reperfusion into the ischemic brain, including oxidative injuries, extravasation of intravascular blood, and recurrent infarctions should be managed appropriately. Statins may be a feasible and effective therapeutic option because of their pleiotropic effects, such as the stabilization of endothelial activation, augmentation of cerebral blood flow, and anti-oxidative and anti-inflammatory effects protecting against ischemic-reperfusion injuries. ${ }^{1-3}$ Several studies have attempted to evaluate therapeutic potential of statins to address this issue, but the majority of such studies analyzed the effect of statin medication before stroke or statin withdrawal after stroke. ${ }^{4}$ Considering the early development of oxidative and inflammatory injuries after recanalization treatment, it may be better to initiate statins earlier to obtain the best effects. Previously, we reported on the efficacy of early statin use after recanalization treatment. ${ }^{5}$ In this current study, we aimed to update the analysis with recent data on recanalization treatment and to determine the subgroups that benefit from early statin initiation ( $\leq 72$ hours).

A total of 857 acute ischemic stroke patients treated with intravenous or endovascular recanalization between July 2007 and December 2015 were identified from a prospective clinical registry from a single center. We retrospectively obtained data on the timing and dose of statins from an electronic barcode medication administration system. Favorable shifts on the modified Rankin Scale score at 3 months and symptomatic hemorrhagic transformation were analyzed, and clustering effects by treatment year were considered. Early statins were used in 67\% $(n=574)$ of the patients, with 33\% $(n=191)$ receiving them within 12 hours. High-intensity statins (atorvastatin $40-80 \mathrm{mg}$ or rosuvastatin $20 \mathrm{mg}$ ) were used in $73 \%$ $(n=418)$ of patients, and low-to-moderate intensity statins were used in 27\% $(n=156)$ of patients (Supplementary Tables 1 and 2). Adjusted multivariable analyses revealed that early statin initiation within 72 hours was associated with a favorable modified Rankin Scale score shift (adjusted odds ratio 1.52; 95\% confidence interval 1.13-2.03) compared with no statin therapy (Figure 1). Early statins were also associated with decreased odds of having symptomatic hemorrhagic transformation (adjusted odds ratio $0.44 ; 95 \%$ confidence interval 0.24-0.83). Early statins showed no substantial exposure-response relationship with time of initiation or intensity. The beneficial effects of early statin initiation maintained in subgroup analyses regarding the mode of recanalization, stroke mechanism, and statin use before the index stroke. The clinical profiles in the exposure-response and subgroup analyses are provided in Supplementary Tables 3-7. The full models of multivariable logistic regression analyses are presented in Supplementary Table 8.

This study shows that early initiation of statins may confer improved functional recovery and decrease hemorrhagic transformation after recanalization treatment for acute ischemic stroke, regardless of treatment modalities, stroke mechanisms, and pre-stroke statin use. Several studies have reported the efficacy of early statin use after recanalization treatment, but 


\begin{tabular}{|c|c|c|c|}
\hline $\mathrm{mRS}$ at $3 \mathrm{mo}$ : favorable shift & & sted OR [95\% Cl] & $P$ \\
\hline Early statin (total) $(n=574)$ & $\longmapsto \vdash$ & $1.52[1.13-2.03]$ & 0.01 \\
\hline$<12$ hours $(n=191)$ & $\longmapsto$ & $1.70[1.17-2.46]$ & 0.01 \\
\hline $12-24$ hours $(n=187)$ & $\longrightarrow$ & $1.48[1.03-2.12]$ & 0.03 \\
\hline $24-72$ hours $(n=196)$ & $\longrightarrow$ & $1.43[1.00-2.03]$ & 0.049 \\
\hline High intensity $(n=418)$ & 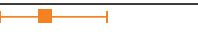 & $1.40[1.02-1.93]$ & 0.04 \\
\hline Low to moderate intensity $(n=156)$ & $\longmapsto$ & $1.78[1.22-2.60]$ & $<0.01$ \\
\hline No early statin $(n=283)$ & & 1.00 [referent] & - \\
\hline
\end{tabular}

\begin{tabular}{|c|c|c|c|c|}
\hline \multicolumn{3}{|c|}{ Symptomatic hemorrhagic transformation } & \multirow{2}{*}{$\begin{array}{r}\text { Adjusted OR [95\% Cl] } \\
0.44[0.24-0.83]\end{array}$} & \multirow{2}{*}{$\begin{array}{c}P \\
0.01\end{array}$} \\
\hline Early statin (total) $(n=574)$ & $\longmapsto$ & & & \\
\hline$<12$ hours $(n=191)$ & $\longmapsto$ & $\longrightarrow$ & $0.73[0.34-1.57]$ & 0.42 \\
\hline $12-24$ hours $(n=187)$ & $\mapsto$ & & $0.19[0.05-0.64]$ & 0.01 \\
\hline $24-72$ hours $(n=196)$ & $\longmapsto$ & & $0.42[0.18-1.02]$ & 0.06 \\
\hline High intensity $(\mathrm{n}=418)$ & $\longmapsto$ & & $0.42[0.21-0.85]$ & 0.02 \\
\hline Low to moderate intensity $(n=156)$ & $\longmapsto$ & $\square$ & $0.49[0.20-1.19]$ & $<0.12$ \\
\hline No early statin $(n=283)$ & & & 1.00 [referent] & - \\
\hline
\end{tabular}

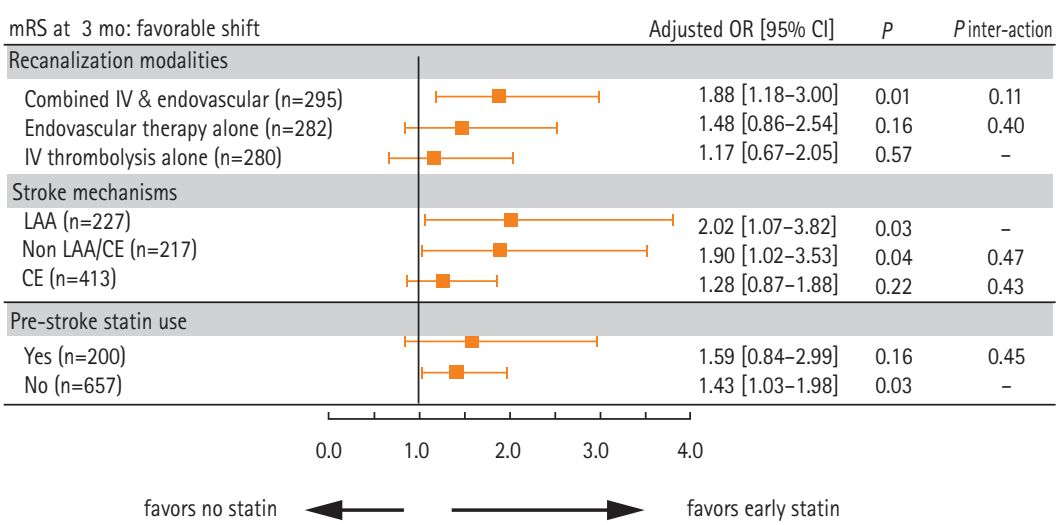

Figure 1. The effects of early statins on functional outcome after recanalization treatment. All models (except symptomatic hemorrhagic transformation) are commonly adjusted with age, sex, premorbid mRS, NIHSS score at arrival, NIHSS score after recanalization treatment, recanalization modalities, stroke mechanism, hypertension, hyperlipidemia, atrial fibrillation, regular smoking, prestroke antithrombotics, prestroke stain, hemoglobin, $\mathrm{HbA} 1 \mathrm{c}$, triglycerides, low density lipoprotein-cholesterol. $\mathrm{mRS}$ at $3 \mathrm{mo}$, modified Rankin Scale at 3 months after stroke; $\mathrm{OR}$, odds ratio; $\mathrm{Cl}$, confidence interval; IV, intravenous; LAA, large artery atherosclerosis; CE, cardioembolism NIHSS, National Institute of Health Stroke Scale. they included only intravenous thrombolysis cases ${ }^{6}$ or were mostly performed before stent-retrievers were used in a majority of patients. ${ }^{5.7}$ In addition, the post-treatment National Institute of Health Stroke Scale score was incorporated into all models in our study as a covariate, which resulted in more accurate assessment of the effect of early statins following recanalization treatment. $^{8}$

\section{Supplementary materials}

Supplementary materials related to this article can be found online at https://doi.org/10.5853/jos.2017.00836.

\section{References}

1. Tsunekawa T, Hayashi T, Kano H, Sumi D, Matsui-Hirai H, Thakur NK, et al. Cerivastatin, a hydroxymethylglutaryl coenzyme a reductase inhibitor, improves endothelial function in elderly diabetic patients within 3 days. Circulation 2001;104:376-379.

2. Ovbiagele B, Saver JL, Starkman S, Kim D, Ali LK, Jahan R, et al. Statin enhancement of collateralization in acute stroke. Neurology 2007;68:2129-2131.

3. Walter DH, Fichtlscherer S, Britten MB, Rosin P, AuchSchwelk W, Schächinger $V_{1}$ et al. Statin therapy, inflammation and recurrent coronary events in patients following coronary stent implantation. J Am Coll Cardiol 2001;38:2006-2012.

4. Hong KS, Lee JS. Statins in acute ischemic stroke: a systematic review. J Stroke 2015;17:282-301.

5. Kang J, Kim N, Park TH, Bang OY, Lee JS, Lee J, et al. Early statin use in ischemic stroke patients treated with recanalization therapy: retrospective observational study. BMC Neurol 2015;15:122.

6. Cappellari M, Bovi P, Moretto G, Zini A, Nencini P, Sessa M, et al. The THRombolysis and STatins (THRaST) study. Neurology 2013;80:655-661.

7. Restrepo L, Bang OY, Ovbiagele B, Ali L, Kim D, Liebeskind DS, et al. Impact of hyperlipidemia and statins on ischemic stroke outcomes after intra-arterial fibrinolysis and percutaneous mechanical embolectomy. Cerebrovasc Dis 2009;28:384-390.

8. Sajobi T, Menon BK, Wang M, Lawal O, Shuaib A, Williams D, 
et al. Early trajectory of stroke severity predicts long-term functional outcomes in ischemic stroke subjects: results from the ESCAPE Trial (endovascular treatment for small core and anterior circulation proximal occlusion with emphasis on minimizing CT to recanalization times). Stroke 2017;48:105110.

\section{Correspondence: Beom Joon Kim}

Department of Neurology, Seoul National University Bundang Hospital, 82 Gumi-ro 173beon-gil, Bundang-gu, Seongnam 13620, Korea

Tel: +82-31-787-7468

Fax: +82-31-787-4563

E-mail:Kim.BJ.Stroke@gmail.com

Received: April 24, 2017

Revised: May 15, 2017

Accepted: June 12, 2017

This study was supported by grant 02-2015-023 from the SNUBH Research Fund.

The authors have no financial conflicts of interest. 
Supplementary Table 1. Clinical characteristics of study patients by early statin use and the timing of statin administration

\begin{tabular}{|c|c|c|c|c|}
\hline Variables & All patients $(n=857)$ & No statins $(n=283)$ & Early statins $(n=574)$ & $P$ \\
\hline \multicolumn{5}{|l|}{ Demographic information } \\
\hline Age & $68.8 \pm 12.6$ & $70.4 \pm 12.9$ & $68.0 \pm 12.4$ & 0.01 \\
\hline Male sex & $494(57.6)$ & $144(50.9)$ & $350(61.0)$ & $<0.01$ \\
\hline Premorbid $\mathrm{mRS}$ score $=0$ & 735 (85.8) & 226 (79.9) & $509(88.7)$ & $<0.01$ \\
\hline Admission year, 2011-2015 & $592(69.1)$ & $158(55.8)$ & $434(75.6)$ & $<0.01$ \\
\hline \multicolumn{5}{|l|}{ Stroke information } \\
\hline NIHSS score at arrival & $12(7-19)$ & $15(9-20)$ & $11(6-18)$ & $<0.01$ \\
\hline Onset to treatment delay (hour) & $2.8(1.7-7.7)$ & $2.8(1.7-6.0)$ & $2.9(1.7-8.6)$ & 0.13 \\
\hline Recanalization treatment & & & & $<0.01$ \\
\hline Intravenous thrombolysis alone & $280(32.7)$ & $71(25.1)$ & $209(36.4)$ & \\
\hline Endovascular therapy alone & $282(32.9)$ & $88(31.1)$ & $194(33.8)$ & \\
\hline Combined & $295(34.4)$ & $124(43.8)$ & $171(29.8)$ & \\
\hline NIHSS score after treatment & $10(4-17)$ & $14(6-20)$ & $8(4-15)$ & $<0.01$ \\
\hline Stroke mechanism & & & & $<0.01$ \\
\hline Large artery atherosclerosis & $227(26.5)$ & $44(15.5)$ & 183 (31.9) & \\
\hline Small vessel occlusion & $33(3.9)$ & $1(0.4)$ & $32(5.6)$ & \\
\hline Cardioembolism & $413(48.2)$ & $172(60.8)$ & $241(42.0)$ & \\
\hline Other determined causes & $41(4.8)$ & $19(6.7)$ & $22(3.8)$ & \\
\hline Undetermined cause & $143(16.7)$ & $47(16.6)$ & $96(16.7)$ & \\
\hline \multicolumn{5}{|l|}{ Vascular risk factors } \\
\hline Hypertension & $579(67.6)$ & $174(61.5)$ & 405 (70.6) & 0.01 \\
\hline Diabetes & $232(27.1)$ & $70(24.7)$ & $162(28.2)$ & 0.28 \\
\hline Hyperlipidemia & $226(26.4)$ & 45 (15.9) & $181(31.5)$ & $<0.01$ \\
\hline Atrial fibrillation & $363(42.4)$ & $158(55.8)$ & $205(35.7)$ & $<0.01$ \\
\hline Regular smoking & $327(38.2)$ & $82(29.0)$ & $245(42.7)$ & $<0.01$ \\
\hline Pre-stroke antithrombotics & $378(44.1)$ & $153(54.1)$ & $225(39.2)$ & $<0.01$ \\
\hline Pre-stroke statins & $200(23.3)$ & $51(18.0)$ & $149(26.0)$ & 0.01 \\
\hline \multicolumn{5}{|l|}{ Laboratory information } \\
\hline White cell count $(\mu \mathrm{L})$ & $8,375 \pm 3,209$ & $8,276 \pm 3,474$ & $8,423 \pm 3,072$ & 0.54 \\
\hline Hemoglobin (g/dL) & $13.6 \pm 2.0$ & $13.4 \pm 2.0$ & $13.7 \pm 2.0$ & 0.02 \\
\hline Initial glucose (mg/dL) & $137 \pm 51$ & $137 \pm 49$ & $137 \pm 51$ & 0.98 \\
\hline $\mathrm{HbA1c}(\%)$ & $6.1 \pm 1.1$ & $6.0 \pm 0.9$ & $6.2 \pm 1.2$ & 0.01 \\
\hline Total cholesterol (mg/dL) & $168 \pm 42$ & $160 \pm 34$ & $172 \pm 44$ & $<0.01$ \\
\hline HDL cholesterol (mg/dL) & $45 \pm 12$ & $46 \pm 13$ & $44 \pm 11$ & 0.14 \\
\hline Triglycerides (mg/dL) & $108 \pm 64$ & $99 \pm 57$ & $112 \pm 67$ & $<0.01$ \\
\hline LDL cholesterol (mg/dL) & $102 \pm 36$ & $95 \pm 29$ & $105 \pm 39$ & $<0.01$ \\
\hline Systolic blood pressure $(\mathrm{mmHg})$ & $152 \pm 27$ & $150 \pm 27$ & $153 \pm 28$ & 0.11 \\
\hline Diastolic blood pressure $(\mathrm{mmHg})$ & $82 \pm 18$ & $81 \pm 16$ & $82 \pm 18$ & 0.19 \\
\hline \multicolumn{5}{|l|}{ Outcome information } \\
\hline mRS score at 3 months & & & & $<0.01$ \\
\hline 0 & $177(20.7)$ & 45 (15.9) & $132(23.0)$ & \\
\hline 1 & $137(16.0)$ & $25(8.8)$ & $112(19.5)$ & \\
\hline 2 & $120(14.0)$ & $36(12.7)$ & $84(14.6)$ & \\
\hline 3 & $116(13.5)$ & $32(11.3)$ & $84(14.6)$ & \\
\hline 4 & $91(10.6)$ & 39 (13.8) & $52(9.1)$ & \\
\hline 5 & $122(14.2)$ & $53(18.7)$ & 69 (12.0) & \\
\hline 6 & $94(11.0)$ & 53 (18.7) & $41(7.1)$ & \\
\hline Symptomatic HT & $52(6.1)$ & $30(10.6)$ & $22(3.8)$ & $<0.01$ \\
\hline
\end{tabular}

Values are presented as mean \pm standard deviation, median (interquartile range), or number (\%). The admission year was divided into 2011-2015 and 20072010 because use of the stent-retriever started in 2011.

mRS, modified Rankin Scale; NIHSS, National Institute of Health Stroke Scale; HDL, high-density lipoprotein; LDL, low-density lipoprotein; HT, hemorrhage transformation. 
Supplementary Table 2. Statin agent, dose, and time of early statin administration

\begin{tabular}{|c|c|c|c|c|}
\hline & Early statins $(n=574)$ & $<12$ hours $(n=191)$ & $12-24$ hours $(n=187)$ & $24-72$ hours $(n=196)$ \\
\hline Atorvastatin (mg) & 541 (94.3) & 187 (97.9) & 179 (95.7) & 175 (89.3) \\
\hline 80 & $313(54.5)$ & 139 (72.8) & $100(53.5)$ & 74 (37.8) \\
\hline 40 & 102 (17.8) & $25(13.1)$ & $42(22.5)$ & 35 (17.9) \\
\hline 20 & $32(5.6)$ & $7(3.7)$ & $12(6.4)$ & $13(6.6)$ \\
\hline 10 & $94(16.4)$ & $16(8.4)$ & $25(13.4)$ & $53(27.0)$ \\
\hline Rosuvastatin (mg) & $12(2.1)$ & $1(0.5)$ & $2(1.1)$ & $9(4.6)$ \\
\hline 20 & $3(0.5)$ & $1(0.5)$ & $1(0.5)$ & $1(0.5)$ \\
\hline 10 & $9(1.6)$ & $0(0.0)$ & $1(0.5)$ & $8(4.1)$ \\
\hline Simvastatin (mg) & $10(1.7)$ & $2(1.0)$ & $4(2.1)$ & $4(2.0)$ \\
\hline 20 & $7(1.2)$ & $2(1.0)$ & $3(1.6)$ & $2(1.0)$ \\
\hline 10 & $3(0.5)$ & $0(0.0)$ & $1(0.5)$ & $2(1.0)$ \\
\hline Pitavastatin (mg) & $6(1.0)$ & $1(0.5)$ & $1(0.5)$ & $4(2.0)$ \\
\hline 2 & $6(1.0)$ & $1(0.5)$ & $1(0.5)$ & $4(2.0)$ \\
\hline Pravastatin (mg) & $4(0.7)$ & $0(0.0)$ & $0(0.0)$ & $4(2.0)$ \\
\hline 40 & $1(0.2)$ & $0(0.0)$ & $0(0.0)$ & $1(0.5)$ \\
\hline 10 & $3(0.5)$ & $0(0.0)$ & $0(0.0)$ & $3(1.5)$ \\
\hline
\end{tabular}

Values are presented as number (\%). High intensity statins were defined as a statin agent and dosage of atorvastatin of $40-80 \mathrm{mg}$ or a dosage of rosuvastatin of $20 \mathrm{mg}$. Low to moderate intensity statins were defined as all other statin agents/doses. 
Supplementary Table 3. Clinical characteristics of study patients by timing of early statin administration

\begin{tabular}{|c|c|c|c|c|c|}
\hline \multirow[b]{2}{*}{ Variables } & \multicolumn{4}{|c|}{ Timing of early statin administration } & \multirow[b]{2}{*}{$P$} \\
\hline & $\begin{array}{l}\text { No statins } \\
(n=283)\end{array}$ & $<12$ hours $(n=191)$ & $12-24$ hours $(n=187)$ & $24-72$ hours $(n=196)$ & \\
\hline \multicolumn{6}{|l|}{ Demographic information } \\
\hline Age & $70.4 \pm 12.9$ & $68.4 \pm 11.9$ & $67.6 \pm 13.0$ & $68.0 \pm 12.5$ & 0.06 \\
\hline Male sex & $144(50.9)$ & $123(64.4)$ & $112(59.9)$ & $115(58.7)$ & 0.03 \\
\hline Premorbid $m R S$ score $=0$ & $226(79.9)$ & $168(88.0)$ & $167(89.3)$ & $174(88.8)$ & 0.01 \\
\hline Admission year, 2011-2015 & $158(55.8)$ & 160 (83.8) & $141(75.4)$ & $133(67.9)$ & $<0.01$ \\
\hline \multicolumn{6}{|l|}{ Stroke information } \\
\hline NIHSS score at arrival & $15(9-20)$ & $11(6-17)$ & $11(6-17)$ & $11.5(6-18)$ & $<0.01$ \\
\hline Onset to treatment delay (hour) & $2.8(1.7-6.0)$ & $3.9(1.8-11.5)$ & $2.9(1.6-6.9)$ & $2.5(1.7-5.9)$ & 0.04 \\
\hline Recanalization treatment & & & & & $<0.01$ \\
\hline Intravenous thrombolysis alone & $71(25.1)$ & 57 (29.8) & $71(38.0)$ & $81(41.3)$ & \\
\hline Endovascular therapy alone & $88(31.1)$ & $87(45.5)$ & $61(32.6)$ & $46(23.5)$ & \\
\hline Combined & $124(43.8)$ & $47(24.6)$ & $55(29.4)$ & 69 (35.2) & \\
\hline NIHSS score after treatment & $14(6-20)$ & $8(4-15)$ & $8(4-13)$ & $8(4-15)$ & $<0.01$ \\
\hline Stroke mechanism & & & & & $<0.01$ \\
\hline Large artery atherosclerosis & 44 (15.5) & 78 (40.8) & $62(33.2)$ & $43(21.9)$ & \\
\hline Small vessel occlusion & $1(0.4)$ & $14(7.3)$ & $8(4.3)$ & $10(5.1)$ & \\
\hline Cardioembolism & $172(60.8)$ & $59(30.9)$ & $78(41.7)$ & $104(53.1)$ & \\
\hline Other determined causes & $19(6.7)$ & $9(4.7)$ & $5(2.7)$ & $8(4.1)$ & \\
\hline Undetermined cause & 47 (16.6) & $31(16.2)$ & 34 (18.2) & 31 (15.8) & \\
\hline \multicolumn{6}{|l|}{ Vascular risk factors } \\
\hline Hypertension & $174(61.5)$ & $138(72.3)$ & $132(70.6)$ & $135(68.9)$ & 0.05 \\
\hline Diabetes & $70(24.7)$ & $60(31.4)$ & $48(25.7)$ & $54(27.6)$ & 0.42 \\
\hline Hyperlipidemia & 45 (15.9) & $57(29.8)$ & 59 (31.6) & 65 (33.2) & $<0.01$ \\
\hline Atrial fibrillation & $158(55.8)$ & $48(25.1)$ & $66(35.3)$ & $91(46.4)$ & $<0.01$ \\
\hline Regular smoking & $82(29.0)$ & $91(47.6)$ & $76(40.6)$ & 78 (39.8) & $<0.01$ \\
\hline Pre-stroke antithrombotics & $153(54.1)$ & $62(32.5)$ & $71(38.0)$ & $92(46.9)$ & $<0.01$ \\
\hline Pre-stroke statins & 51 (18.0) & $42(22.0)$ & $47(25.1)$ & $60(30.6)$ & 0.01 \\
\hline \multicolumn{6}{|l|}{ Laboratory information } \\
\hline White cell count $(\mu \mathrm{L})$ & $8,276 \pm 3,474$ & $8,572 \pm 2,868$ & $8,375 \pm 3,274$ & $8,324 \pm 3,075$ & 0.79 \\
\hline Hemoglobin (g/dL) & $13.4 \pm 2.0$ & $13.9 \pm 2.0$ & $13.6 \pm 2.0$ & $13.6 \pm 2.0$ & 0.05 \\
\hline Initial glucose (mg/dL) & $137 \pm 49$ & $141 \pm 58$ & $133 \pm 49$ & $136 \pm 46$ & 0.48 \\
\hline $\mathrm{HbA1c}(\%)$ & $6.0 \pm 0.9$ & $6.2 \pm 1.2$ & $6.2 \pm 1.1$ & $6.3 \pm 1.1$ & 0.07 \\
\hline Total cholesterol (mg/dL) & $160 \pm 34$ & $172 \pm 49$ & $167 \pm 42$ & $176 \pm 41$ & $<0.01$ \\
\hline HDL cholesterol (mg/dL) & $46 \pm 13$ & $43 \pm 11$ & $44 \pm 11$ & $46 \pm 11$ & 0.04 \\
\hline Triglycerides (mg/dL) & $99 \pm 57$ & $116 \pm 68$ & $111 \pm 72$ & $109 \pm 60$ & 0.03 \\
\hline LDL cholesterol (mg/dL) & $95 \pm 29$ & $106 \pm 41$ & $101 \pm 36$ & $108 \pm 38$ & $<0.01$ \\
\hline Systolic blood pressure (mmHg) & $150 \pm 27$ & $155 \pm 28$ & $153 \pm 29$ & $151 \pm 26$ & 0.21 \\
\hline Diastolic blood pressure $(\mathrm{mmHg})$ & $81 \pm 16$ & $84 \pm 18$ & $83 \pm 20$ & $81 \pm 18$ & 0.22 \\
\hline \multicolumn{6}{|l|}{ Outcome information } \\
\hline mRS score at 3 months & & & & & $<0.01$ \\
\hline 0 & 45 (15.9) & $47(24.6)$ & $42(22.5)$ & $43(21.9)$ & \\
\hline 1 & $25(8.8)$ & $36(18.8)$ & $33(17.6)$ & $43(21.9)$ & \\
\hline 2 & $36(12.7)$ & $27(14.1)$ & $34(18.2)$ & $23(11.7)$ & \\
\hline 3 & $32(11.3)$ & $27(14.1)$ & $25(13.4)$ & $32(16.3)$ & \\
\hline 4 & 39 (13.8) & $15(7.9)$ & $18(9.6)$ & $19(9.7)$ & \\
\hline 5 & $53(18.7)$ & $24(12.6)$ & $21(11.2)$ & $24(12.2)$ & \\
\hline 6 & $53(18.7)$ & $15(7.9)$ & $14(7.5)$ & $12(6.1)$ & \\
\hline Symptomatic HT & $30(10.6)$ & $12(6.3)$ & $3(1.6)$ & $7(3.6)$ & $<0.01$ \\
\hline
\end{tabular}

Values are presented as mean \pm standard deviation, median (interquartile range), or number (\%).

mRS, modified Rankin Scale; NIHSS, National Institute of Health Stroke Scale; HDL, high-density lipoprotein; LDL, low-density lipoprotein; HT, hemorrhage transformation. 
Supplementary Table 4. Clinical profiles by statin intensity

\begin{tabular}{|c|c|c|c|c|}
\hline \multirow{2}{*}{ Variables } & \multirow{2}{*}{ No statins $(n=283)$} & \multicolumn{2}{|c|}{ Intensity of early statins } & \multirow{2}{*}{$P$} \\
\hline & & Low to moderate $(n=156)$ & High $(n=418)$ & \\
\hline \multicolumn{5}{|l|}{ Demographic information } \\
\hline Age & $70.4 \pm 12.9$ & $68.7 \pm 12.3$ & $67.8 \pm 12.5$ & 0.02 \\
\hline Male sex & $144(50.9)$ & $92(59.0)$ & $258(61.7)$ & 0.02 \\
\hline Premorbid $\mathrm{mRS}$ score $=0$ & 226 (79.9) & $138(88.5)$ & 371 (88.8) & $<0.01$ \\
\hline Admission year, 2011-2015 & $158(55.8)$ & $99(63.5)$ & $335(80.1)$ & $<0.01$ \\
\hline \multicolumn{5}{|l|}{ Stroke information } \\
\hline NIHSS score at arrival & $15(9-20)$ & $12(6-18)$ & $11(6-17)$ & $<0.01$ \\
\hline Onset to treatment delay (hour) & $2.8(1.7-6.0)$ & $2.8(1.7-6.7)$ & $3.0(1.7-9.0)$ & 0.32 \\
\hline Recanalization treatment & & & & $<0.01$ \\
\hline Intravenous thrombolysis alone & $71(25.1)$ & $67(42.9)$ & $142(34.0)$ & \\
\hline Endovascular therapy alone & $88(31.1)$ & $46(29.5)$ & $148(35.4)$ & \\
\hline Combined & $124(43.8)$ & $43(27.6)$ & $128(30.6)$ & \\
\hline NIHSS score after treatment & $14(6-20)$ & $8(4-15.25)$ & $8(4-14)$ & $<0.01$ \\
\hline Stroke mechanism & & & & $<0.01$ \\
\hline Large artery atherosclerosis & $44(15.5)$ & $38(24.4)$ & $145(34.7)$ & \\
\hline Small vessel occlusion & $1(0.4)$ & $10(6.4)$ & $22(5.3)$ & \\
\hline Cardioembolism & $172(60.8)$ & $87(55.8)$ & $154(36.8)$ & \\
\hline Other determined cause & $19(6.7)$ & $4(2.6)$ & $18(4.3)$ & \\
\hline Undetermined cause & 47 (16.6) & $17(10.9)$ & 79 (18.9) & \\
\hline \multicolumn{5}{|l|}{ Vascular risk factors } \\
\hline Hypertension & $174(61.5)$ & $107(68.6)$ & $298(71.3)$ & 0.02 \\
\hline Diabetes & $70(24.7)$ & $40(25.6)$ & $122(29.2)$ & 0.39 \\
\hline Hyperlipidemia & $45(15.9)$ & $58(37.2)$ & $123(29.4)$ & $<0.01$ \\
\hline Atrial fibrillation & $158(55.8)$ & $77(49.4)$ & $128(30.6)$ & $<0.01$ \\
\hline Regular smoking & $82(29.0)$ & 68 (43.6) & $177(42.3)$ & $<0.01$ \\
\hline Pre-stroke antithrombotics & $153(54.1)$ & $82(52.6)$ & $143(34.2)$ & $<0.01$ \\
\hline Pre-stroke statins & 51 (18.0) & $67(42.9)$ & 82 (19.6) & $<0.01$ \\
\hline \multicolumn{5}{|l|}{ Laboratory information } \\
\hline White cell count $(\mu \mathrm{L})$ & $8,276 \pm 3,474$ & $8,458 \pm 2,772$ & $8,410 \pm 3,179$ & 0.81 \\
\hline Hemoglobin (g/dL) & $13.4 \pm 2.0$ & $14.0 \pm 1.9$ & $13.6 \pm 2.0$ & 0.01 \\
\hline Initial glucose (mg/dL) & $137 \pm 49$ & $140 \pm 50$ & $136 \pm 52$ & 0.62 \\
\hline $\mathrm{HbA} 1 \mathrm{c}(\%)$ & $6.0 \pm 0.9$ & $6.3 \pm 1.2$ & $6.2 \pm 1.2$ & 0.02 \\
\hline Total cholesterol (mg/dL) & $160 \pm 34$ & $171 \pm 43$ & $172 \pm 45$ & $<0.01$ \\
\hline HDL cholesterol (mg/dL) & $46 \pm 13$ & $45 \pm 11$ & $44 \pm 11$ & 0.14 \\
\hline Triglycerides (mg/dL) & $99 \pm 57$ & $114 \pm 73$ & $111 \pm 64$ & 0.02 \\
\hline LDL cholesterol (mg/dL) & $95 \pm 29$ & $103 \pm 39$ & $106 \pm 38$ & $<0.01$ \\
\hline Systolic blood pressure (mmHg) & $150 \pm 27$ & $152 \pm 26$ & $153 \pm 28$ & 0.24 \\
\hline Diastolic blood pressure $(\mathrm{mmHg})$ & $81 \pm 16$ & $80 \pm 19$ & $83 \pm 18$ & 0.06 \\
\hline \multicolumn{5}{|l|}{ Outcome information } \\
\hline $\mathrm{mRS}$ score at 3 months & & & & $<0.01$ \\
\hline 0 & 45 (15.9) & $38(24.4)$ & $94(22.5)$ & \\
\hline 1 & $25(8.8)$ & $34(21.8)$ & 78 (18.7) & \\
\hline 2 & $36(12.7)$ & $24(15.4)$ & $60(14.4)$ & \\
\hline 3 & $32(11.3)$ & $21(13.5)$ & $63(15.1)$ & \\
\hline 4 & 39 (13.8) & $14(9.0)$ & $38(9.1)$ & \\
\hline 5 & $53(18.7)$ & $15(9.6)$ & $54(12.9)$ & \\
\hline 6 & $53(18.7)$ & $10(6.4)$ & $31(7.4)$ & \\
\hline Symptomatic HT & $30(10.6)$ & $7(4.5)$ & $15(3.6)$ & $<0.01$ \\
\hline
\end{tabular}

Values are presented as mean \pm standard deviation, median (interquartile range), or number (\%).

mRS, modified Rankin Scale; NIHSS, National Institute of Health Stroke Scale; HDL, high-density lipoprotein; LDL, low-density lipoprotein; HT, hemorrhage transformation. 
Supplementary Table 5. Clinical profiles of subgroups divided by recanalization modalities

\begin{tabular}{|c|c|c|c|c|c|c|c|c|c|}
\hline \multirow[b]{2}{*}{ Variables } & \multicolumn{3}{|c|}{ Combined IV-endovascular } & \multicolumn{3}{|c|}{ Endovascular only } & \multicolumn{3}{|c|}{ IV only } \\
\hline & $\begin{array}{l}\text { No statins } \\
(n=124)\end{array}$ & $\begin{array}{c}\text { Early statins } \\
(n=171)\end{array}$ & $P$ & $\begin{array}{l}\text { No statins } \\
(\mathrm{n}=88)\end{array}$ & $\begin{array}{l}\text { Early statins } \\
(n=194)\end{array}$ & $P$ & $\begin{array}{l}\text { No statins } \\
(n=71)\end{array}$ & $\begin{array}{l}\text { Early statins } \\
\qquad(\mathrm{n}=209)\end{array}$ & $P$ \\
\hline \multicolumn{10}{|l|}{ Demographic information } \\
\hline Age & $70.6 \pm 12.6$ & $68.8 \pm 12.4$ & 0.22 & $69.5 \pm 11.9$ & $69.0 \pm 11.5$ & 0.71 & $71.2 \pm 14.4$ & $66.4 \pm 13.1$ & 0.02 \\
\hline Male sex & $60(48.4)$ & $111(64.9)$ & $<0.01$ & $49(55.7)$ & $114(58.8)$ & 0.63 & $35(49.3)$ & $125(59.8)$ & 0.12 \\
\hline Premorbid $m R S$ score $=0$ & $106(85.5)$ & $149(87.1)$ & 0.68 & 65 (73.9) & $169(87.1)$ & 0.01 & 55 (77.5) & $191(91.4)$ & $<0.01$ \\
\hline Admission year, 2011-2015 & $69(55.6)$ & $135(78.9)$ & $<0.01$ & $51(58.0)$ & $157(80.9)$ & $<0.01$ & $38(53.5)$ & $142(67.9)$ & 0.03 \\
\hline \multicolumn{10}{|l|}{ Stroke information } \\
\hline NIHSS score at arrival & $16(12-20)$ & $15(10-20)$ & 0.16 & $16(10-21)$ & $12(6-18)$ & 0.01 & $10(5.5-17)$ & $8(5-12)$ & 0.04 \\
\hline Onset to treatment delay (hour) & $2.0(1.4-2.9)$ & $1.9(1.3-2.9)$ & 0.23 & $7.0(4.6-11.6)$ & $10.0(6.2-14.7)$ & 0.51 & $2.7(1.8-3.2)$ & $2.1(1.5-3.2)$ & 0.57 \\
\hline Recanalization treatment & & & - & & & - & & & - \\
\hline Intravenous thrombolysis alone & $0(0)$ & $0(0)$ & & $0(0)$ & $0(0)$ & & $71(100)$ & $209(100)$ & \\
\hline Endovascular therapy alone & $0(0)$ & $0(0)$ & & $88(100)$ & $194(100)$ & & $0(0)$ & $0(0)$ & \\
\hline Combined & $124(100)$ & $171(100)$ & & $0(0)$ & $0(0)$ & & $0(0)$ & $0(0)$ & \\
\hline NIHSS score after treatment & $14(6-20)$ & $10(5-16)$ & $<0.01$ & $17(9-21)$ & $10.5(5-16)$ & $<0.01$ & $9(4-17)$ & $5(4-11)$ & $<0.01$ \\
\hline Stroke mechanism & & & $<0.01$ & & & 0.01 & & & $<0.01$ \\
\hline Large artery atherosclerosis & 14 (11.3) & $41(24.0)$ & & 17 (19.3) & $73(37.6)$ & & 13 (18.3) & $69(33.0)$ & \\
\hline Small vessel occlusion & $0(0)$ & $0(0)$ & & $0(0)$ & $0(0)$ & & $1(1.4)$ & $32(15.3)$ & \\
\hline Cardioembolism & $91(73.4)$ & $94(55.0)$ & & $45(51.1)$ & 79 (40.7) & & $36(50.7)$ & $68(32.5)$ & \\
\hline Other determined cause & $6(4.8)$ & $5(2.9)$ & & 9 (10.2) & $33(17.0)$ & & $4(5.6)$ & 8 (3.8) & \\
\hline Undetermined cause & $13(10.5)$ & $31(18.1)$ & & $17(19.3)$ & $33(17.0)$ & & $17(23.9)$ & $32(15.3)$ & \\
\hline \multicolumn{10}{|l|}{ Vascular risk factors } \\
\hline Hypertension & $75(60.5)$ & $129(75.4)$ & 0.01 & $53(60.2)$ & $142(73.2)$ & 0.03 & $46(64.8)$ & $134(64.1)$ & 0.92 \\
\hline Diabetes & $33(26.6)$ & $49(28.7)$ & 0.7 & $15(17.0)$ & $59(30.4)$ & 0.02 & $22(31.0)$ & $54(25.8)$ & 0.40 \\
\hline Hyperlipidemia & $21(16.9)$ & $58(33.9)$ & $<0.01$ & $13(14.8)$ & $58(29.9)$ & 0.01 & $11(15.5)$ & $65(31.1)$ & 0.01 \\
\hline Atrial fibrillation & $82(66.1)$ & $85(49.7)$ & $<0.01$ & $41(46.6)$ & $69(35.6)$ & 0.08 & 35 (49.3) & $51(24.4)$ & $<0.01$ \\
\hline Regular smoking & $31(25.0)$ & $68(39.8)$ & 0.01 & $31(35.2)$ & $88(45.4)$ & 0.11 & $20(28.2)$ & $89(42.6)$ & 0.03 \\
\hline Pre-stroke antithrombotics & $73(58.9)$ & $78(45.6)$ & 0.02 & $42(47.7)$ & $81(41.8)$ & 0.35 & $38(53.5)$ & $66(31.6)$ & $<0.01$ \\
\hline Pre-stroke statins & $22(17.7)$ & $51(29.8)$ & 0.02 & $15(17.0)$ & $44(22.7)$ & 0.28 & $14(19.7)$ & $54(25.8)$ & 0.30 \\
\hline \multicolumn{10}{|l|}{ Laboratory information } \\
\hline White cell count $(\mu \mathrm{L})$ & $7,778 \pm 2,842$ & $8,181 \pm 2,763$ & 0.22 & $8,545 \pm 3,834$ & $8,901 \pm 3,120$ & 0.45 & $8,811 \pm 3,915$ & $8,179 \pm 3,225$ & 0.22 \\
\hline Hemoglobin (g/dL) & $13.5 \pm 1.7$ & $13.6 \pm 1.8$ & 0.49 & $13.2 \pm 2.6$ & $13.6 \pm 2.2$ & 0.24 & $13.4 \pm 1.8$ & $13.9 \pm 1.9$ & 0.06 \\
\hline Initial glucose (mg/dL) & $139 \pm 55$ & $130 \pm 37$ & 0.09 & $129 \pm 31$ & $142 \pm 57$ & 0.01 & $142 \pm 55$ & $138 \pm 55$ & 0.60 \\
\hline $\mathrm{HbA} 1 \mathrm{c}(\%)$ & $6.1 \pm 0.9$ & $6.1 \pm 0.8$ & 0.76 & $5.8 \pm 0.6$ & $6.1 \pm 1.3$ & $<0.01$ & $6.2 \pm 1.1$ & $6.2 \pm 1.3$ & 0.85 \\
\hline Total cholesterol (mg/dL) & $163 \pm 37$ & $165 \pm 45$ & 0.74 & $154 \pm 34$ & $169 \pm 44$ & $<0.01$ & $162 \pm 29$ & $180 \pm 43$ & $<0.01$ \\
\hline HDL cholesterol (mg/dL) & $46 \pm 14$ & $44 \pm 11$ & 0.12 & $44 \pm 12$ & $44 \pm 12$ & 0.68 & $46 \pm 12$ & $45 \pm 11$ & 0.53 \\
\hline Triglycerides (mg/dL) & $94 \pm 63$ & $108 \pm 62$ & 0.07 & $103 \pm 54$ & $109 \pm 62$ & 0.42 & $103 \pm 48$ & $118 \pm 74$ & 0.06 \\
\hline LDL cholesterol (mg/dL) & $98 \pm 28$ & $99 \pm 40$ & 0.78 & $90 \pm 29$ & $104 \pm 37$ & $<0.01$ & $96 \pm 28$ & $111 \pm 38$ & $<0.01$ \\
\hline Systolic blood pressure (mmHg) & $148 \pm 28$ & $148 \pm 25$ & 0.92 & $147 \pm 27$ & $151 \pm 26$ & 0.26 & $156 \pm 26$ & $159 \pm 29$ & 0.39 \\
\hline Diastolic blood pressure $(\mathrm{mmHg})$ & $81 \pm 18$ & $79 \pm 16$ & 0.29 & $79 \pm 15$ & $81 \pm 17$ & 0.51 & $82 \pm 15$ & $87 \pm 21$ & 0.04 \\
\hline \multicolumn{10}{|l|}{ Outcome information } \\
\hline $\mathrm{mRS}$ score at 3 months & & & $<0.01$ & & & $<0.01$ & & & 0.18 \\
\hline 0 & $20(16.1)$ & $44(25.7)$ & & $8(9.1)$ & $31(16.0)$ & & $17(23.9)$ & $57(27.3)$ & \\
\hline 1 & $12(9.7)$ & $29(17.0)$ & & $4(4.5)$ & $35(18.0)$ & & $9(12.7)$ & $48(23.0)$ & \\
\hline 2 & $14(11.3)$ & $27(15.8)$ & & $12(13.6)$ & $30(15.5)$ & & $10(14.1)$ & $27(12.9)$ & \\
\hline 3 & $11(8.9)$ & $21(12.3)$ & & $13(14.8)$ & $33(17.0)$ & & $8(11.3)$ & $30(14.4)$ & \\
\hline 4 & $18(14.5)$ & $11(6.4)$ & & $15(17.0)$ & $23(11.9)$ & & $6(8.5)$ & $18(8.6)$ & \\
\hline 5 & $28(22.6)$ & $28(16.4)$ & & $16(18.2)$ & 25 (12.9) & & $9(12.7)$ & $16(7.7)$ & \\
\hline 6 & $21(16.9)$ & $11(6.4)$ & & $20(22.7)$ & 17 (8.8) & & 12 (16.9) & $13(6.2)$ & \\
\hline Symptomatic HT & 14 (11.3) & $5(2.9)$ & $<0.01$ & $11(12.5)$ & $10(5.2)$ & 0.03 & $5(7.0)$ & $7(3.3)$ & 0.18 \\
\hline
\end{tabular}

Values are presented as mean \pm standard deviation, median (interquartile range), or number (\%).

IV, intravenous; mRS, modified Rankin Scale; NIHSS, National Institute of Health Stroke Scale; HDL, high-density lipoprotein; LDL, low-density lipoprotein; HT, hemorrhage transformation. 
Supplementary Table 6. Clinical profiles of subgroups by stroke mechanism

\begin{tabular}{|c|c|c|c|c|c|c|c|c|c|}
\hline \multirow[b]{2}{*}{ Variables } & \multicolumn{3}{|c|}{ LAA } & \multicolumn{3}{|c|}{ CE } & \multicolumn{3}{|c|}{ Non-LAA/CE } \\
\hline & $\begin{array}{c}\text { No statins } \\
(n=44)\end{array}$ & $\begin{array}{l}\text { Early statins } \\
\quad(n=183)\end{array}$ & $P$ & $\begin{array}{c}\text { No statins } \\
(n=172)\end{array}$ & $\begin{array}{l}\text { Early statins } \\
\quad(n=241)\end{array}$ & $P$ & $\begin{array}{l}\text { No statins } \\
(n=67)\end{array}$ & $\begin{array}{c}\text { Early statins } \\
\quad(n=150)\end{array}$ & $P$ \\
\hline \multicolumn{10}{|l|}{ Demographic information } \\
\hline Age & $67.6 \pm 11.4$ & $66.8 \pm 11.7$ & 0.67 & $73.0 \pm 11.8$ & $71.2 \pm 10.5$ & 0.10 & $65.7 \pm 14.8$ & $64.4 \pm 14.7$ & 0.56 \\
\hline Male sex & $29(65.9)$ & $130(71.0)$ & 0.51 & $83(48.3)$ & $123(51.0)$ & 0.58 & $32(47.8)$ & $97(64.7)$ & 0.02 \\
\hline Premorbid mRS score $=0$ & $35(79.5)$ & 169 (92.3) & 0.01 & $140(81.4)$ & $205(85.1)$ & 0.32 & $51(76.1)$ & $135(90.0)$ & 0.01 \\
\hline Admission year, 2011-2015 & $15(34.1)$ & $127(69.4)$ & $<0.01$ & $107(62.2)$ & $188(78.0)$ & $<0.01$ & $36(53.7)$ & $119(79.3)$ & $<0.01$ \\
\hline \multicolumn{10}{|l|}{ Stroke information } \\
\hline NIHSS score at arrival & $11(6.75-19.25)$ & $10(5-15)$ & 0.05 & $16(10.75-20)$ & $15(9-20)$ & 0.13 & $14(6-19)$ & $9(5-15)$ & $<0.01$ \\
\hline Onset to treatment delay (hour) & $3.6(2.4-11.3)$ & $3.8(1.9-11.2)$ & 0.31 & $2.6(1.7-5.3)$ & $2.6(1.7-8.1)$ & 0.01 & $2.7(1.7-5.5)$ & $2.5(1.4-5.5)$ & 0.33 \\
\hline Recanalization treatment & & & 0.37 & & & 0.02 & & & 0.10 \\
\hline Intravenous thrombolysis alone & $13(29.5)$ & $69(37.7)$ & & $36(20.9)$ & $68(28.2)$ & & $22(32.8)$ & $72(48.0)$ & \\
\hline Endovascular therapy alone & $17(38.6)$ & 73 (39.9) & & 45 (26.2) & 79 (32.8) & & $26(38.8)$ & $42(28.0)$ & \\
\hline Combined & $14(31.8)$ & $41(22.4)$ & & $91(52.9)$ & $94(39.0)$ & & $19(28.4)$ & $36(24.0)$ & \\
\hline NIHSS score after treatment & $11(6-19)$ & $6(4-13)$ & 0.01 & $15(6-21)$ & $11(5-17)$ & $<0.01$ & $14(4.5-19.5)$ & $6(4-11)$ & $<0.01$ \\
\hline Stroke mechanism & & & - & & & - & & & $<0.01$ \\
\hline Large artery atherosclerosis & $44(100)$ & $183(100)$ & & $0(0)$ & $0(0)$ & & $0(0)$ & $0(0)$ & \\
\hline Small vessel occlusion & $0(0)$ & $0(0)$ & & $0(0)$ & $0(0)$ & & $1(1.5)$ & $32(21.3)$ & \\
\hline Cardioembolism & $0(0)$ & $0(0)$ & & $172(100)$ & $241(100)$ & & $0(0)$ & $0(0)$ & \\
\hline Other determined cause & $0(0)$ & $0(0)$ & & $0(0)$ & $0(0)$ & & $19(28.4)$ & $22(14.7)$ & \\
\hline Undetermined cause & $0(0)$ & $0(0)$ & & $0(0)$ & $0(0)$ & & $47(70.1)$ & $96(64.0)$ & \\
\hline \multicolumn{10}{|l|}{ Vascular risk factors } \\
\hline Hypertension & $30(68.2)$ & $131(71.6)$ & 0.66 & $106(61.6)$ & $176(73.0)$ & 0.02 & $38(56.7)$ & $98(65.3)$ & 0.23 \\
\hline Diabetes & $13(29.5)$ & $56(30.6)$ & 0.89 & $42(24.4)$ & $71(29.5)$ & 0.26 & $15(22.4)$ & $35(23.3)$ & 0.88 \\
\hline Hyperlipidemia & 8 (18.2) & $52(28.4)$ & 0.17 & $26(15.1)$ & 81 (33.6) & $<0.01$ & $11(16.4)$ & $48(32.0)$ & 0.02 \\
\hline Atrial fibrillation & $1(2.3)$ & $0(0)$ & 0.04 & $147(85.5)$ & $194(80.5)$ & 0.19 & $10(14.9)$ & $11(7.3)$ & 0.08 \\
\hline Regular smoking & $19(43.2)$ & $99(54.1)$ & 0.19 & $39(22.7)$ & $77(32.0)$ & 0.04 & $24(35.8)$ & $69(46.0)$ & 0.16 \\
\hline Pre-stroke antithrombotics & $17(38.6)$ & $59(32.2)$ & 0.42 & $108(62.8)$ & $129(53.5)$ & 0.06 & $28(41.8)$ & $37(24.7)$ & 0.01 \\
\hline Pre-stroke statins & $10(22.7)$ & 40 (21.9) & 0.90 & $31(18.0)$ & $76(31.5)$ & $<0.01$ & $10(14.9)$ & $33(22.0)$ & 0.23 \\
\hline \multicolumn{10}{|l|}{ Laboratory information } \\
\hline White cell count ( $\mu \mathrm{L})$ & $9,058 \pm 3,568$ & $8,932 \pm 3,288$ & 0.83 & $7,752 \pm 2,948$ & $7,976 \pm 2,949$ & 0.45 & $9,105 \pm 4,350$ & $8,521 \pm 2,902$ & 0.32 \\
\hline Hemoglobin (g/dL) & $14.2 \pm 1.7$ & $14.0 \pm 2.0$ & 0.56 & $13.5 \pm 1.9$ & $13.5 \pm 1.9$ & 0.73 & $12.5 \pm 2.3$ & $13.7 \pm 2.2$ & $<0.01$ \\
\hline Initial glucose (mg/dL) & $145 \pm 51$ & $146 \pm 62$ & 0.85 & $134 \pm 44$ & $132 \pm 45$ & 0.64 & $140 \pm 59$ & $134 \pm 45$ & 0.47 \\
\hline $\mathrm{HbA1c}(\%)$ & $6.1 \pm 0.8$ & $6.4 \pm 1.4$ & 0.11 & $6.0 \pm 0.9$ & $6.2 \pm 1.0$ & 0.08 & $5.9 \pm 1.1$ & $6.1 \pm 1.0$ & 0.36 \\
\hline Total cholesterol (mg/dL) & $180 \pm 35$ & $172 \pm 43$ & 0.17 & $155 \pm 31$ & $168 \pm 44$ & $<0.01$ & $161 \pm 37$ & $178 \pm 46$ & 0.01 \\
\hline HDL cholesterol (mg/dL) & $48 \pm 16$ & $43 \pm 11$ & 0.10 & $47 \pm 12$ & $45 \pm 12$ & 0.21 & $42 \pm 12$ & $44 \pm 10$ & 0.18 \\
\hline Triglycerides (mg/dL) & $117 \pm 56$ & $118 \pm 74$ & 0.88 & $88 \pm 41$ & $101 \pm 59$ & 0.01 & $116 \pm 80$ & $121 \pm 67$ & 0.67 \\
\hline LDL cholesterol (mg/dL) & $109 \pm 29$ & $105 \pm 37$ & 0.37 & $91 \pm 27$ & $103 \pm 37$ & $<0.01$ & $96 \pm 29$ & $110 \pm 42$ & 0.01 \\
\hline Systolic blood pressure (mmHg) & $148 \pm 27$ & $156 \pm 28$ & 0.11 & $151 \pm 27$ & $151 \pm 24$ & 0.79 & $147 \pm 26$ & $153 \pm 31$ & 0.10 \\
\hline Diastolic blood pressure $(\mathrm{mmHg})$ & $82 \pm 15$ & $83 \pm 18$ & 0.67 & $81 \pm 18$ & $81 \pm 19$ & 0.83 & $80 \pm 13$ & $84 \pm 18$ & 0.11 \\
\hline \multicolumn{10}{|l|}{ Outcome information } \\
\hline $\mathrm{mRS}$ score at 3 months & & & 0.03 & & & 0.06 & & & $<0.01$ \\
\hline 0 & $4(9.1)$ & $38(20.8)$ & & $29(16.9)$ & $55(22.8)$ & & $12(17.9)$ & $39(26.0)$ & \\
\hline 1 & $4(9.1)$ & $43(23.5)$ & & $17(9.9)$ & $35(14.5)$ & & $4(6.0)$ & $34(22.7)$ & \\
\hline 2 & $7(15.9)$ & $29(15.8)$ & & $23(13.4)$ & $30(12.4)$ & & $6(9.0)$ & $28(18.7)$ & \\
\hline 3 & $7(15.9)$ & $29(15.8)$ & & $17(9.9)$ & $34(14.1)$ & & $8(11.9)$ & $21(14.0)$ & \\
\hline 4 & $7(15.9)$ & $17(9.3)$ & & $25(14.5)$ & $27(11.2)$ & & $7(10.4)$ & $8(5.3)$ & \\
\hline 5 & $9(20.5)$ & $20(10.9)$ & & $32(18.6)$ & $40(16.6)$ & & $12(17.9)$ & $9(6.0)$ & \\
\hline 6 & $6(13.6)$ & $10(5.5)$ & & 29 (16.9) & $20(8.3)$ & & 18 (26.9) & $11(7.3)$ & \\
\hline Symptomatic HT & $6(13.6)$ & $5(2.7)$ & $<0.01$ & $18(10.5)$ & $12(5.0)$ & 0.03 & $6(9.0)$ & $5(3.3)$ & 0.08 \\
\hline
\end{tabular}

Values are presented as mean \pm standard deviation, median (interquartile range), or number (\%).

LAA, large artery atherosclerosis; CE, cardioembolism; mRS, modified Rankin Scale; NIHSS, National Institute of Health Stroke Scale; HDL, high-density lipoprotein; LDL, low-density lipoprotein; HT, hemorrhage transformation. 
Supplementary Table 7. Clinical profiles of subgroups by pre-stroke statin use

\begin{tabular}{|c|c|c|c|c|c|c|}
\hline \multirow{2}{*}{ Variables } & \multicolumn{3}{|c|}{ Pre-stroke statin use: yes } & \multicolumn{3}{|c|}{ Pre-stroke statin use: no } \\
\hline & No statins $(n=51)$ & Early statins $(n=149)$ & $P$ & No statins $(n=232)$ & Early statins $(n=425)$ & $P$ \\
\hline \multicolumn{7}{|l|}{ Demographic information } \\
\hline Age & $73.1 \pm 9.3$ & $70.9 \pm 10.4$ & 0.15 & $69.9 \pm 13.5$ & $67.0 \pm 12.9$ & 0.01 \\
\hline Male sex & $25(49.0)$ & $86(57.7)$ & 0.28 & 119 (51.3) & $264(62.1)$ & 0.01 \\
\hline Premorbid $\mathrm{mRS}$ score $=0$ & $41(80.4)$ & $122(81.9)$ & 0.81 & 185 (79.7) & $387(91.1)$ & $<0.01$ \\
\hline Admission year, 2011-2015 & $32(62.7)$ & $117(78.5)$ & 0.03 & $126(54.3)$ & $317(74.6)$ & $<0.01$ \\
\hline \multicolumn{7}{|l|}{ Stroke information } \\
\hline NIHSS score at arrival & $15(8-19)$ & $12(6-18)$ & 0.21 & $15(9-20)$ & $11(6-17)$ & $<0.01$ \\
\hline Onset to treatment delay (hour) & $3.2(1.8-6.4)$ & $2.8(1.6-7.9)$ & 0.92 & $2.7(1.7-5.8)$ & $2.9(1.7-8.8)$ & 0.06 \\
\hline Recanalization treatment & & & 0.43 & & & $<0.01$ \\
\hline Intravenous thrombolysis alone & $14(27.5)$ & $54(36.2)$ & & $57(24.6)$ & $155(36.5)$ & \\
\hline Endovascular therapy alone & $15(29.4)$ & $44(29.5)$ & & $73(31.5)$ & $150(35.3)$ & \\
\hline Combined & $22(43.1)$ & $51(34.2)$ & & $102(44.0)$ & $120(28.2)$ & \\
\hline NIHSS score after treatment & $15(6.5-18.5)$ & $9(4-16)$ & 0.01 & $14(5.75-20)$ & $8(4-14)$ & $<0.01$ \\
\hline Stroke mechanism & & & 0.45 & & & $<0.01$ \\
\hline Large artery atherosclerosis & $10(19.6)$ & $40(26.8)$ & & 34 (14.7) & $143(33.6)$ & \\
\hline Small vessel occlusion & $0(0)$ & $6(4.0)$ & & $1(0.4)$ & $26(6.1)$ & \\
\hline Cardioembolism & $31(60.8)$ & 76 (51.0) & & $141(60.8)$ & $165(38.8)$ & \\
\hline Other determined cause & $2(3.9)$ & $4(2.7)$ & & $17(7.3)$ & $18(4.2)$ & \\
\hline Undetermined cause & $8(15.7)$ & $23(15.4)$ & & 39 (16.8) & 73 (17.2) & \\
\hline \multicolumn{7}{|l|}{ Vascular risk factors } \\
\hline Hypertension & $43(84.3)$ & $125(83.9)$ & 0.94 & $131(56.5)$ & $280(65.9)$ & 0.02 \\
\hline Diabetes & 15 (29.4) & $66(44.3)$ & 0.06 & $55(23.7)$ & $96(22.6)$ & 0.75 \\
\hline Hyperlipidemia & $34(66.7)$ & $131(87.9)$ & $<0.01$ & $11(4.7)$ & 50 (11.8) & $<0.01$ \\
\hline Atrial fibrillation & $25(49.0)$ & $66(44.3)$ & 0.56 & $133(57.3)$ & $139(32.7)$ & $<0.01$ \\
\hline Regular smoking & $12(23.5)$ & $56(37.6)$ & 0.07 & 70 (30.2) & $189(44.5)$ & $<0.01$ \\
\hline Pre-stroke antithrombotics & $40(78.4)$ & $108(72.5)$ & 0.40 & $113(48.7)$ & $117(27.5)$ & $<0.01$ \\
\hline Pre-stroke statins & $51(100)$ & $149(100)$ & - & $0(0)$ & $0(0)$ & - \\
\hline \multicolumn{7}{|l|}{ Laboratory information } \\
\hline White cell count $(\mu \mathrm{L})$ & $8,038 \pm 2,564$ & $8,004 \pm 2,940$ & 0.94 & $8,328 \pm 3,646$ & $8,570 \pm 3,107$ & 0.39 \\
\hline Hemoglobin (g/dL) & $13.3 \pm 2.1$ & $13.4 \pm 1.8$ & 0.83 & $13.4 \pm 2.0$ & $13.8 \pm 2.1$ & 0.01 \\
\hline Initial glucose (mg/dL) & $141 \pm 55$ & $141 \pm 51$ & 0.96 & $136 \pm 48$ & $135 \pm 52$ & 0.89 \\
\hline $\mathrm{HbA} 1 \mathrm{c}(\%)$ & $6.2 \pm 1.3$ & $6.5 \pm 1.2$ & 0.28 & $6.0 \pm 0.8$ & $6.1 \pm 1.1$ & 0.03 \\
\hline Total cholesterol (mg/dL) & $157 \pm 32$ & $152 \pm 41$ & 0.40 & $161 \pm 35$ & $179 \pm 43$ & $<0.01$ \\
\hline HDL cholesterol (mg/dL) & $46 \pm 12$ & $44 \pm 10$ & 0.26 & $45 \pm 13$ & $44 \pm 11$ & 0.28 \\
\hline Triglycerides (mg/dL) & $101 \pm 54$ & $104 \pm 53$ & 0.71 & $99 \pm 57$ & $115 \pm 70$ & $<0.01$ \\
\hline LDL cholesterol (mg/dL) & $90 \pm 29$ & $87 \pm 37$ & 0.51 & $96 \pm 28$ & $111 \pm 37$ & $<0.01$ \\
\hline Systolic blood pressure (mmHg) & $157 \pm 24$ & $152 \pm 27$ & 0.22 & $148 \pm 28$ & $153 \pm 28$ & 0.02 \\
\hline Diastolic blood pressure $(\mathrm{mmHg})$ & $81 \pm 14$ & $80 \pm 16$ & 0.94 & $81 \pm 17$ & $83 \pm 19$ & 0.11 \\
\hline \multicolumn{7}{|l|}{ Outcome information } \\
\hline $\mathrm{mRS}$ score at 3 months & & & $<0.01$ & & & $<0.01$ \\
\hline 0 & $6(11.8)$ & $45(30.2)$ & & 39 (16.8) & $87(20.5)$ & \\
\hline 1 & $3(5.9)$ & $23(15.4)$ & & $22(9.5)$ & 89 (20.9) & \\
\hline 2 & $7(13.7)$ & $19(12.8)$ & & $29(12.5)$ & $65(15.3)$ & \\
\hline 3 & 5 (9.8) & $17(11.4)$ & & $27(11.6)$ & $67(15.8)$ & \\
\hline 4 & $13(25.5)$ & $12(8.1)$ & & $26(11.2)$ & $40(9.4)$ & \\
\hline 5 & $9(17.6)$ & 19 (12.8) & & $44(19.0)$ & $50(11.8)$ & \\
\hline 6 & $8(15.7)$ & $14(9.4)$ & & 45 (19.4) & $27(6.4)$ & \\
\hline Symptomatic HT & $9(17.6)$ & $4(2.7)$ & $<0.01$ & $21(9.1)$ & $18(4.2)$ & 0.01 \\
\hline
\end{tabular}

Values are presented as mean \pm standard deviation, median (interquartile range), or number (\%).

mRS, modified Rankin Scale; NIHSS, National Institute of Health Stroke Scale; HDL, high-density lipoprotein; LDL, low-density lipoprotein; HT, hemorrhage transformation. 
Supplementary Table 8. Full models of multivariable analyses

\begin{tabular}{|c|c|c|}
\hline Variables & Adjusted OR (95\% Cl) & $P$ \\
\hline \multicolumn{3}{|l|}{ All patients: mRS shift at 3 months* } \\
\hline Early statins & $1.52(1.13-2.03)$ & 0.01 \\
\hline Age (per 1-year increase) & $0.96(0.95-0.97)$ & $<0.01$ \\
\hline Male sex & $0.77(0.56-1.05)$ & 0.10 \\
\hline Premorbid $\mathrm{mRS}$ score $=0$ & $1.68(1.15-2.46)$ & 0.01 \\
\hline NIHSS score at arrival (per 1-point increase) & $1.03(1.00-1.06)$ & 0.03 \\
\hline Onset to treatment delay (per 1-hour increase) & $1.00(0.99-1.01)$ & 0.82 \\
\hline \multicolumn{3}{|l|}{ Recanalization treatment } \\
\hline Intravenous thrombolysis alone & Referent & - \\
\hline Endovascular therapy alone & $0.86(0.62-1.21)$ & 0.40 \\
\hline Combined & $1.02(0.74-1.42)$ & 0.89 \\
\hline NIHSS score after treatment (per 1-point increase) & $0.82(0.79-0.84)$ & $<0.01$ \\
\hline \multicolumn{3}{|l|}{ Stroke mechanism } \\
\hline Large artery atherosclerosis & Referent & - \\
\hline Small vessel occlusion & $0.91(0.46-1.80)$ & 0.78 \\
\hline Cardioembolism & $1.52(0.97-2.39)$ & 0.07 \\
\hline Other determined cause & $0.33(0.17-0.65)$ & $<0.01$ \\
\hline Undetermined cause & $1.01(0.68-1.49)$ & 0.97 \\
\hline Hypertension & $1.26(0.95-1.68)$ & 0.11 \\
\hline Hyperlipidemia & $0.98(0.67-1.45)$ & 0.93 \\
\hline Atrial fibrillation & $0.87(0.57-1.32)$ & 0.51 \\
\hline Regular smoking & $1.41(1.031 .92)$ & 0.03 \\
\hline Pre-stroke antithrombotics & $0.90(0.67-1.20)$ & 0.47 \\
\hline Pre-stroke statins & $1.16(0.76-1.76)$ & 0.49 \\
\hline Hemoglobin (per $1 \mathrm{~g} / \mathrm{dL}$ increase) & $1.06(0.98-1.14)$ & 0.14 \\
\hline HbA1c (per 1\% increase) & $0.87(0.77-0.97)$ & 0.02 \\
\hline Triglycerides (per 1 mg/dL increase) & $1.001(0.999-1.003)$ & 0.54 \\
\hline LDL cholesterol (per $1 \mathrm{mg} / \mathrm{dL}$ increase) & 0.995 (0.991-0.998) & 0.01 \\
\hline \multicolumn{3}{|l|}{ All patients: $m R S 0-1$ at 3 months ${ }^{+}$} \\
\hline Early statins & $1.66(1.09-2.52)$ & 0.02 \\
\hline Age & $0.64(0.52-0.80)$ & $<0.01$ \\
\hline Male sex & $0.75(0.47-1.19)$ & 0.22 \\
\hline Premorbid mRS score $=0$ & $1.88(1.01-3.49)$ & 0.047 \\
\hline NIHSS score at arrival (per 1-point increase) & $1.02(0.98-1.06)$ & 0.35 \\
\hline Onset to treatment delay (per 1-hour increase) & $0.988(0.971-1.005)$ & 0.16 \\
\hline \multicolumn{3}{|l|}{ Recanalization treatment } \\
\hline Intravenous thrombolysis alone & Referent & \\
\hline Endovascular therapy alone & $0.64(0.40-1.02)$ & 0.06 \\
\hline Combined & $0.87(0.55-1.38)$ & 0.56 \\
\hline NIHSS score after treatment (per 1-point increase) & $0.82(0.78-0.85)$ & $<0.01$ \\
\hline \multicolumn{3}{|l|}{ Stroke mechanism } \\
\hline Large artery atherosclerosis & Referent & \\
\hline Small vessel occlusion & $0.62(0.26-1.50)$ & 0.29 \\
\hline Cardioembolism & $1.54(0.82-2.92)$ & 0.18 \\
\hline Other determined cause & $0.78(0.31-1.96)$ & 0.60 \\
\hline Undetermined cause & $1.01(0.59-1.72)$ & 0.98 \\
\hline Hypertension & $1.10(0.73-1.64)$ & 0.65 \\
\hline Hyperlipidemia & $1.50(0.85-2.65)$ & 0.16 \\
\hline Atrial fibrillation & $1.01(0.56-1.84)$ & 0.96 \\
\hline Regular smoking & $1.98(1.27-3.08)$ & $<0.01$ \\
\hline
\end{tabular}


Supplementary Table 8. Continued

\begin{tabular}{|c|c|c|}
\hline Variables & Adjusted OR (95\% Cl) & $P$ \\
\hline Pre-stroke antithrombotics & $0.84(0.55-1.27)$ & 0.40 \\
\hline Pre-stroke statins & $0.96(0.51-1.78)$ & 0.89 \\
\hline Hemoglobin (per $1 \mathrm{~g} / \mathrm{dL}$ increase) & $1.03(0.93-1.14)$ & 0.54 \\
\hline HbA1c (per 1\% increase) & $0.86(0.72-1.02)$ & 0.08 \\
\hline Triglycerides & $1.02(0.85-1.21)$ & 0.87 \\
\hline LDL cholesterol & $0.77(0.64-0.92)$ & $<0.01$ \\
\hline \multicolumn{3}{|l|}{ All patients: symptomatic HT } \\
\hline Early statins & $0.44(0.240 .83)$ & 0.01 \\
\hline Age (per 1-year increase) & $1.00(0.98-1.03)$ & 0.89 \\
\hline Male sex & $1.27(0.69-2.35)$ & 0.44 \\
\hline Premorbid mRS score $=0$ & $1.13(0.51-2.52)$ & 0.76 \\
\hline NIHSS score at arrival (per 1-point increase) & $0.93(0.89-0.99)$ & 0.01 \\
\hline Onset to treatment delay (per 1-hour increase) & $1.00(0.98-1.03)$ & 0.87 \\
\hline \multicolumn{3}{|l|}{ Recanalization treatment } \\
\hline Intravenous thrombolysis alone & Referent & - \\
\hline Endovascular therapy alone & $1.27(0.56-2.90)$ & 0.57 \\
\hline Combined & $1.17(0.53-2.61)$ & 0.69 \\
\hline NIHSS score after treatment (per 1-point increase) & $1.13(1.07-1.18)$ & $<0.01$ \\
\hline \multicolumn{3}{|c|}{ Exposure (time of statin administration)-response analysis: $\mathrm{mRS}$ shift at 3 months $^{\dagger}$} \\
\hline Statin time: $<12$ hours & $1.70(1.172 .46)$ & 0.01 \\
\hline Statin time: $12-24$ hours & $1.48(1.03-2.12)$ & 0.03 \\
\hline Statin time: $24-72$ hours & $1.43(1.001-2.03)$ & 0.049 \\
\hline Age (per 1-year increase) & $0.96(0.95-0.97)$ & $<0.01$ \\
\hline Male sex & $0.77(0.56-1.05)$ & 0.10 \\
\hline Premorbid $m R S$ score $=0$ & $1.69(1.16-2.47)$ & 0.01 \\
\hline NIHSS score at arrival (per 1-point increase) & $1.03(1.002-1.06)$ & 0.03 \\
\hline Onset to treatment delay (per 1-hour increase) & $1.00(0.99-1.01)$ & 0.81 \\
\hline \multicolumn{3}{|l|}{ Recanalization treatment } \\
\hline Intravenous thrombolysis alone & Referent & - \\
\hline Endovascular therapy alone & $0.84(0.60-1.18)$ & 0.32 \\
\hline Combined & $1.01(0.73-1.41)$ & 0.95 \\
\hline NIHSS score after treatment (per 1-point increase) & $0.82(0.80-0.84)$ & $<0.01$ \\
\hline \multicolumn{3}{|l|}{ Stroke mechanism } \\
\hline Large artery atherosclerosis & Referent & \\
\hline Small vessel occlusion & $0.89(0.45-1.76)$ & 0.73 \\
\hline Cardioembolism & $1.53(0.98-2.41)$ & 0.06 \\
\hline Other determined cause & $0.33(0.17-0.66)$ & $<0.01$ \\
\hline Undetermined cause & $1.01(0.68-1.49)$ & 0.96 \\
\hline Hypertension & $1.26(0.95-1.67)$ & 0.12 \\
\hline Hyperlipidemia & $0.98(0.66-1.44)$ & 0.91 \\
\hline Atrial fibrillation & $0.88(0.58-1.34)$ & 0.55 \\
\hline Regular smoking & $1.41(1.03-1.92)$ & 0.03 \\
\hline Pre-stroke antithrombotics & $0.91(0.68-1.21)$ & 0.51 \\
\hline Pre-stroke statins & $1.17(0.77-1.78)$ & 0.47 \\
\hline Hemoglobin (per $1 \mathrm{~g} / \mathrm{dL}$ increase) & $1.06(0.98-1.14)$ & 0.16 \\
\hline HbA1c (per 1\% increase) & $0.87(0.77-0.97)$ & 0.02 \\
\hline Triglycerides (per 1 mg/dL increase) & $1.001(0.999-1.003)$ & 0.53 \\
\hline LDL cholesterol (per 1 mg/dL increase) & $0.995(0.991-0.998)$ & 0.01 \\
\hline
\end{tabular}


Supplementary Table 8. Continued

\begin{tabular}{|c|c|c|}
\hline Variables & Adjusted OR (95\% Cl) & $P$ \\
\hline \multicolumn{3}{|c|}{ Exposure (time of statin administration)-response analysis: mRS 0-1 at 3 months $^{+}$} \\
\hline Statin time: $<12$ hours & $1.91(1.13-3.24)$ & 0.02 \\
\hline Statin time: $12-24$ hours & $1.39(0.84-2.32)$ & 0.20 \\
\hline Statin time: $24-72$ hours & $1.74(1.06-2.86)$ & 0.03 \\
\hline Age & $0.64(0.51-0.79)$ & $<0.01$ \\
\hline Male sex & $0.74(0.47-1.18)$ & 0.21 \\
\hline Premorbid $\mathrm{mRS}$ score $=0$ & $1.88(1.01-3.50)$ & 0.045 \\
\hline NIHSS score at arrival (per 1-point increase) & $1.02(0.98-1.06)$ & 0.38 \\
\hline Onset to treatment delay (per 1-hour increase) & $0.99(0.97-1.01)$ & 0.17 \\
\hline \multicolumn{3}{|l|}{ Recanalization treatment } \\
\hline Intravenous thrombolysis alone & Referent & \\
\hline Endovascular therapy alone & $0.62(0.39-1.01)$ & 0.053 \\
\hline Combined & $0.86(0.55-1.36)$ & 0.52 \\
\hline NIHSS score after treatment (per 1-point increase) & $0.82(0.78-0.85)$ & $<0.01$ \\
\hline \multicolumn{3}{|l|}{ Stroke mechanism } \\
\hline Large artery atherosclerosis & Referent & \\
\hline Small vessel occlusion & $0.59(0.24-1.44)$ & 0.25 \\
\hline Cardioembolism & $1.53(0.81-2.90)$ & 0.19 \\
\hline Other determined cause & $0.75(0.30-1.89)$ & 0.55 \\
\hline Undetermined cause & $1.00(0.59-1.72)$ & 0.99 \\
\hline Hypertension & $1.10(0.73-1.64)$ & 0.65 \\
\hline Hyperlipidemia & $1.51(0.85-2.65)$ & 0.16 \\
\hline Atrial fibrillation & $1.02(0.56-1.86)$ & 0.94 \\
\hline Regular smoking & $1.97(1.26-3.06)$ & $<0.01$ \\
\hline Pre-stroke antithrombotics & $0.84(0.55-1.27)$ & 0.40 \\
\hline Pre-stroke statins & $0.95(0.51-1.79)$ & 0.89 \\
\hline Hemoglobin (per $1 \mathrm{~g} / \mathrm{dL}$ increase) & $1.03(0.93-1.14)$ & 0.55 \\
\hline $\mathrm{HbA} 1 \mathrm{c}$ (per 1\% increase) & $0.86(0.72-1.02)$ & 0.08 \\
\hline Triglycerides & $1.01(0.85-1.21)$ & 0.88 \\
\hline LDL cholesterol & $0.76(0.63-0.91)$ & $<0.01$ \\
\hline \multicolumn{3}{|c|}{ Exposure (time of statin administration)-response analysis: symptomatic HT } \\
\hline Statin time: $<12$ hours & $0.73(0.34-1.57)$ & 0.42 \\
\hline Statin time: $12-24$ hours & $0.19(0.05-0.64)$ & 0.01 \\
\hline Statin time: $24-72$ hours & $0.42(0.18-1.02)$ & 0.06 \\
\hline Age (per 1-year increase) & $1.00(0.98-1.03)$ & 0.93 \\
\hline Male sex & $1.26(0.68-2.33)$ & 0.47 \\
\hline Premorbid mRS score $=0$ & $1.13(0.50-2.52)$ & 0.77 \\
\hline NIHSS score at arrival (per 1-point increase) & $0.94(0.89-0.99)$ & 0.02 \\
\hline Onset to treatment delay (per 1-hour increase) & $1.00(0.98-1.03)$ & 0.84 \\
\hline \multicolumn{3}{|l|}{ Recanalization treatment } \\
\hline Intravenous thrombolysis alone & Referent & \\
\hline Endovascular therapy alone & $1.16(0.51-2.68)$ & 0.72 \\
\hline Combined & $1.17(0.53-2.61)$ & 0.70 \\
\hline NIHSS score after treatment (per 1-point increase) & $1.12(1.07-1.18)$ & $<0.01$ \\
\hline \multicolumn{3}{|c|}{ Exposure (statin intensity)-response analysis: $\mathrm{mRS}$ shift at 3 months ${ }^{\S}$} \\
\hline Statins: low to moderate intensity & $1.78(1.22-2.60)$ & $<0.01$ \\
\hline Statins: high intensity & $1.40(1.02-1.93)$ & 0.04 \\
\hline Age (per 1-year increase) & $0.96(0.95-0.97)$ & $<0.01$ \\
\hline
\end{tabular}


Supplementary Table 8. Continued

\begin{tabular}{|c|c|c|}
\hline Variables & Adjusted OR (95\% Cl) & $P$ \\
\hline Male sex & $0.77(0.56-1.06)$ & 0.11 \\
\hline Premorbid $m R S$ score $=0$ & $1.69(1.15-2.46)$ & 0.01 \\
\hline NIHSS score at arrival (per 1-point increase) & $1.03(1.003-1.06)$ & 0.03 \\
\hline Onset to treatment delay (per 1-hour increase) & $1.00(0.99-1.01)$ & 0.79 \\
\hline \multicolumn{3}{|l|}{ Recanalization treatment } \\
\hline Intravenous thrombolysis alone & Referent & \\
\hline Endovascular therapy alone & $0.88(0.63-1.23)$ & 0.45 \\
\hline Combined & $1.04(0.75-1.45)$ & 0.81 \\
\hline NIHSS score after treatment (per 1-point increase) & $0.82(0.79-0.84)$ & $<0.01$ \\
\hline \multicolumn{3}{|l|}{ Stroke mechanism } \\
\hline Large artery atherosclerosis & Referent & \\
\hline Small vessel occlusion & $0.91(0.46-1.80)$ & 0.78 \\
\hline Cardioembolism & $1.50(0.96-2.36)$ & 0.08 \\
\hline Other determined cause & $0.32(0.16-0.64)$ & $<0.01$ \\
\hline Undetermined cause & $1.01(0.68-1.48)$ & 0.98 \\
\hline Hypertension & $1.28(0.96-1.70)$ & 0.09 \\
\hline Hyperlipidemia & $1.01(0.68-1.50)$ & 0.96 \\
\hline Atrial fibrillation & $0.85(0.56-1.29)$ & 0.45 \\
\hline Regular smoking & $1.39(1.02-1.91)$ & 0.04 \\
\hline Pre-stroke antithrombotics & $0.90(0.67-1.19)$ & 0.45 \\
\hline Pre-stroke statins & $1.09(0.71-1.67)$ & 0.71 \\
\hline Hemoglobin (per $1 \mathrm{~g} / \mathrm{dL}$ increase) & $1.06(0.98-1.14)$ & 0.15 \\
\hline HbA1c (per 1\% increase) & $0.87(0.77-0.97)$ & 0.02 \\
\hline Triglycerides (per 1 mg/dL increase) & $1.001(0.999-1.002)$ & 0.60 \\
\hline LDL cholesterol (per 1 mg/dL increase) & $0.995(0.991-0.998)$ & 0.01 \\
\hline \multicolumn{3}{|c|}{ Exposure (statin intensity)-response analysis: mRS 0-1 at 3 months $^{+}$} \\
\hline Statins: low to moderate intensity & $2.04(1.20-3.47)$ & 0.01 \\
\hline Statins: high intensity & $1.51(0.97-2.35)$ & 0.07 \\
\hline Age & $0.64(0.51-0.79)$ & $<0.01$ \\
\hline Male sex & $0.76(0.48-1.21)$ & 0.25 \\
\hline Premorbid mRS score $=0$ & $1.90(1.02-3.54)$ & 0.04 \\
\hline NIHSS score at arrival (per 1-point increase) & $1.02(0.98-1.06)$ & 0.37 \\
\hline Onset to treatment delay (per 1-hour increase) & $0.99(0.97-1.005)$ & 0.16 \\
\hline \multicolumn{3}{|l|}{ Recanalization treatment } \\
\hline Intravenous thrombolysis alone & Referent & \\
\hline Endovascular therapy alone & $0.65(0.41-1.04)$ & 0.07 \\
\hline Combined & $0.89(0.57-1.41)$ & 0.62 \\
\hline NIHSS score after treatment (per 1-point increase) & $0.82(0.78-0.85)$ & $<0.01$ \\
\hline \multicolumn{3}{|l|}{ Stroke mechanism } \\
\hline Large artery atherosclerosis & Referent & \\
\hline Small vessel occlusion & $0.61(0.25-1.48)$ & 0.28 \\
\hline Cardioembolism & $1.51(0.80-2.86)$ & 0.21 \\
\hline Other determined cause & $0.76(0.30-1.91)$ & 0.56 \\
\hline Undetermined cause & $1.01(0.59-1.72)$ & 0.97 \\
\hline Hypertension & $1.12(0.75-1.68)$ & 0.58 \\
\hline Hyperlipidemia & $1.56(0.89-2.76)$ & 0.12 \\
\hline Atrial fibrillation & $0.99(0.54-1.79)$ & 0.97 \\
\hline Regular smoking & $1.96(1.26-3.05)$ & $<0.01$ \\
\hline Pre-stroke antithrombotics & $0.83(0.55-1.25)$ & 0.37 \\
\hline
\end{tabular}


Supplementary Table 8. Continued

\begin{tabular}{|c|c|c|}
\hline Variables & Adjusted OR $(95 \% \mathrm{Cl})$ & $P$ \\
\hline Pre-stroke statins & $0.88(0.46-1.66)$ & 0.69 \\
\hline Hemoglobin (per $1 \mathrm{~g} / \mathrm{dL}$ increase) & $1.03(0.93-1.14)$ & 0.62 \\
\hline HbA1c (per 1\% increase) & $0.86(0.72-1.02)$ & 0.08 \\
\hline Triglycerides & $1.01(0.85-1.20)$ & 0.92 \\
\hline LDL cholesterol & $0.76(0.64-0.92)$ & $<0.01$ \\
\hline \multicolumn{3}{|c|}{ Exposure (statin intensity)-response analysis: symptomatic HT } \\
\hline Statins: low to moderate intensity & $0.49(0.20-1.19)$ & 0.12 \\
\hline Statins: high intensity & $0.42(0.21-0.85)$ & 0.02 \\
\hline Age (per 1-year increase) & $1.00(0.98-1.03)$ & 0.90 \\
\hline Male sex & $1.27(0.69-2.34)$ & 0.44 \\
\hline Premorbid $m R S$ score $=0$ & $1.13(0.51-2.52)$ & 0.77 \\
\hline NIHSS score at arrival (per 1-point increase) & $0.93(0.89-0.99)$ & 0.01 \\
\hline Onset to treatment delay (per 1-hour increase) & $1.00(0.98-1.03)$ & 0.87 \\
\hline \multicolumn{3}{|l|}{ Recanalization treatment } \\
\hline Intravenous thrombolysis alone & Referent & \\
\hline Endovascular therapy alone & $1.28(0.56-2.93)$ & 0.56 \\
\hline Combined & $1.18(0.53-2.63)$ & 0.68 \\
\hline NIHSS score after treatment (per 1-point increase) & $1.13(1.07-1.18)$ & $<0.01$ \\
\hline \multicolumn{3}{|c|}{ Subgroup (combined IV-endovascular treatment): mRS shift at 3 months" } \\
\hline Early statins & $1.88(1.18-3.00)$ & 0.01 \\
\hline Age (per 1-year increase) & $0.96(0.94-0.98)$ & $<0.01$ \\
\hline Male sex & $0.41(0.24-0.72)$ & $<0.01$ \\
\hline Premorbid $\mathrm{mRS}$ score $=0$ & $1.05(0.55-2.04)$ & 0.88 \\
\hline NIHSS score at arrival (per 1-point increase) & $1.06(1.01-1.11)$ & 0.01 \\
\hline Onset to treatment delay (per 1-hour increase) & $1.02(0.98-1.06)$ & 0.42 \\
\hline NIHSS score after treatment (per 1-point increase) & $0.80(0.77-0.84)$ & $<0.01$ \\
\hline \multicolumn{3}{|l|}{ Stroke mechanism } \\
\hline Large artery atherosclerosis & Referent & - \\
\hline Cardioembolism & $3.27(1.34-8.01)$ & 0.01 \\
\hline Other determined cause & $0.45(0.12-1.67)$ & 0.23 \\
\hline Undetermined cause & $1.89(0.88-4.05)$ & 0.10 \\
\hline Hypertension & $1.40(0.82-2.39)$ & 0.22 \\
\hline Hyperlipidemia & $0.83(0.41-1.68)$ & 0.61 \\
\hline Atrial fibrillation & $0.79(0.37-1.69)$ & 0.54 \\
\hline Regular smoking & $2.00(1.15-3.45)$ & 0.01 \\
\hline Pre-stroke antithrombotics & $0.77(0.47-1.27)$ & 0.30 \\
\hline Pre-stroke statins & $1.25(0.59-2.63)$ & 0.56 \\
\hline Hemoglobin (per $1 \mathrm{~g} / \mathrm{dL}$ increase) & $1.19(1.02-1.39)$ & 0.03 \\
\hline $\mathrm{HbA1c}$ (per 1\% increase) & $0.85(0.66-1.10)$ & 0.22 \\
\hline Triglycerides (per 1 mg/dL increase) & $1.003(0.999-1.007)$ & 0.12 \\
\hline LDL cholesterol (per 1 mg/dL increase) & $0.995(0.988-1.001)$ & 0.12 \\
\hline \multicolumn{3}{|c|}{ Subgroup (combined IV-endovascular treatment): mRS 0-1 at 3 months ${ }^{\dagger}$} \\
\hline Early statins & $2.28(1.05-4.94)$ & 0.04 \\
\hline Age & $0.72(0.47-1.09)$ & 0.12 \\
\hline Male sex & $0.18(0.07-0.47)$ & $<0.01$ \\
\hline Premorbid mRS score $=0$ & $1.19(0.39-3.63)$ & 0.76 \\
\hline NIHSS score at arrival (per 1-point increase) & $1.03(0.97-1.09)$ & 0.40 \\
\hline Onset to treatment delay (per 1-hour increase) & $1.04(0.97-1.11)$ & 0.24 \\
\hline NIHSS score after treatment (per 1-point increase) & $0.77(0.72-0.83)$ & $<0.01$ \\
\hline
\end{tabular}


Supplementary Table 8. Continued

\begin{tabular}{|c|c|c|}
\hline Variables & Adjusted OR (95\% Cl) & $P$ \\
\hline \multicolumn{3}{|l|}{ Stroke mechanism } \\
\hline Large artery atherosclerosis & Referent & - \\
\hline Cardioembolism & $7.02(1.49-32.95)$ & 0.01 \\
\hline Other determined cause & $3.02(0.52-17.46)$ & 0.22 \\
\hline Undetermined cause & $3.94(1.19-13.04)$ & 0.02 \\
\hline Hypertension & $0.82(0.35-1.91)$ & 0.64 \\
\hline Hyperlipidemia & $2.52(0.69-9.24)$ & 0.16 \\
\hline Atrial fibrillation & $1.00(0.28-3.58)$ & 0.995 \\
\hline Regular smoking & 3.75 (1.61-8.78) & $<0.01$ \\
\hline Pre-stroke antithrombotics & $0.59(0.26-1.37)$ & 0.22 \\
\hline Pre-stroke statins & $0.60(0.14-2.50)$ & 0.48 \\
\hline Hemoglobin (per $1 \mathrm{~g} / \mathrm{dL}$ increase) & $1.35(1.05-1.74)$ & 0.02 \\
\hline HbA1c (per 1\% increase) & $0.91(0.61-1.36)$ & 0.65 \\
\hline Triglycerides & $1.61(1.04-2.51)$ & 0.03 \\
\hline LDL cholesterol & $0.63(0.44-0.90)$ & 0.01 \\
\hline \multicolumn{3}{|c|}{$\begin{array}{l}\text { Subgroup (combined IV-endovascular treatment): symptom- } \\
\text { atic HT }\end{array}$} \\
\hline Early statins & $0.29(0.09-0.87)$ & 0.03 \\
\hline Age (per 1-year increase) & $1.01(0.96-1.06)$ & 0.68 \\
\hline Male sex & $1.70(0.58-4.98)$ & 0.33 \\
\hline Premorbid mRS score $=0$ & $1.56(0.31-7.79)$ & 0.59 \\
\hline NIHSS score at arrival (per 1-point increase) & $0.96(0.87-1.05)$ & 0.39 \\
\hline Onset to treatment delay (per 1-hour increase) & $1.03(0.98-1.09)$ & 0.27 \\
\hline NIHSS score after treatment (per 1-point increase) & $1.12(1.04-1.21)$ & $<0.01$ \\
\hline \multicolumn{3}{|l|}{ Subgroup (endovascular only): mRS shift at 3 months" } \\
\hline Early statins & $1.48(0.86-2.54)$ & 0.16 \\
\hline Age (per 1-year increase) & $0.96(0.94-0.99)$ & $<0.01$ \\
\hline Male sex & $0.83(0.47-1.47)$ & 0.52 \\
\hline Premorbid $m R S$ score $=0$ & $2.62(1.41-4.90)$ & $<0.01$ \\
\hline NIHSS score at arrival (per 1-point increase) & $1.02(0.98-1.07)$ & 0.31 \\
\hline Onset to treatment delay (per 1-hour increase) & $1.01(0.99-1.02)$ & 0.45 \\
\hline NIHSS score after treatment (per 1-point increase) & $0.82(0.78-0.86)$ & $<0.01$ \\
\hline \multicolumn{3}{|l|}{ Stroke mechanism } \\
\hline Large artery atherosclerosis & Referent & - \\
\hline Cardioembolism & $0.92(0.41-2.07)$ & 0.84 \\
\hline Other determined cause & $0.21(0.06-0.73)$ & 0.01 \\
\hline Undetermined cause & $0.69(0.35-1.36)$ & 0.28 \\
\hline Hypertension & $1.03(0.62-1.72)$ & 0.90 \\
\hline Hyperlipidemia & $1.46(0.75-2.85)$ & 0.27 \\
\hline Atrial fibrillation & $0.76(0.35-1.66)$ & 0.49 \\
\hline Regular smoking & $1.03(0.58-1.82)$ & 0.91 \\
\hline Pre-stroke antithrombotics & $1.02(0.60-1.71)$ & 0.95 \\
\hline Pre-stroke statins & $1.07(0.51-2.24)$ & 0.87 \\
\hline Hemoglobin (per $1 \mathrm{~g} / \mathrm{dL}$ increase) & $1.11(0.98-1.26)$ & 0.10 \\
\hline HbA1c (per 1\% increase) & $0.82(0.66-1.02)$ & 0.08 \\
\hline Triglycerides (per 1 mg/dL increase) & $1.001(0.997-1.004)$ & 0.77 \\
\hline LDL cholesterol (per 1 mg/dL increase) & $0.996(0.989-1.003)$ & 0.25 \\
\hline \multicolumn{3}{|l|}{ Subgroup (endovascular only): mRS 0-1 at 3 months $^{+}$} \\
\hline Early statins & $1.92(0.80-4.59)$ & 0.14 \\
\hline Age & $0.63(0.41-0.95)$ & 0.03 \\
\hline
\end{tabular}


Supplementary Table 8. Continued

\begin{tabular}{|c|c|c|}
\hline Variables & Adjusted OR $(95 \% \mathrm{Cl})$ & $P$ \\
\hline Male sex & $0.85(0.33-2.17)$ & 0.73 \\
\hline Premorbid $\mathrm{mRS}$ score $=0$ & $3.14(0.84-11.74)$ & 0.09 \\
\hline NIHSS score at arrival (per 1-point increase) & $0.99(0.92-1.06)$ & 0.71 \\
\hline Onset to treatment delay (per 1-hour increase) & $0.98(0.96-1.01)$ & 0.19 \\
\hline NIHSS score after treatment (per 1-point increase) & $0.84(0.77-0.90)$ & $<0.01$ \\
\hline \multicolumn{3}{|l|}{ Stroke mechanism } \\
\hline Large artery atherosclerosis & Referent & - \\
\hline Cardioembolism & $0.83(0.24-2.89)$ & 0.77 \\
\hline Other determined cause & $0.46(0.08-2.71)$ & 0.39 \\
\hline Undetermined cause & $0.45(0.17-1.19)$ & 0.11 \\
\hline Hypertension & $0.96(0.43-2.14)$ & 0.92 \\
\hline Hyperlipidemia & $1.57(0.57-4.35)$ & 0.39 \\
\hline Atrial fibrillation & $1.00(0.29-3.40)$ & 0.997 \\
\hline Regular smoking & $1.34(0.55-3.27)$ & 0.52 \\
\hline Pre-stroke antithrombotics & $1.15(0.51-2.60)$ & 0.74 \\
\hline Pre-stroke statins & $1.42(0.44-4.59)$ & 0.56 \\
\hline Hemoglobin (per $1 \mathrm{~g} / \mathrm{dL}$ increase) & $1.02(0.85-1.23)$ & 0.81 \\
\hline $\mathrm{HbA1c}$ (per 1\% increase) & $1.08(0.78-1.49)$ & 0.63 \\
\hline Triglycerides & $1.02(0.74-1.40)$ & 0.90 \\
\hline LDL cholesterol & $0.83(0.58-1.18)$ & 0.29 \\
\hline \multicolumn{3}{|l|}{ Subgroup (endovascular only): symptomatic HT } \\
\hline Early statins & $0.44(0.16-1.19)$ & 0.11 \\
\hline Age (per 1-year increase) & $0.97(0.93-1.01)$ & 0.14 \\
\hline Male sex & $0.62(0.23-1.67)$ & 0.35 \\
\hline Premorbid $\mathrm{mRS}$ score $=0$ & $1.78(0.46-6.83)$ & 0.40 \\
\hline NIHSS score at arrival (per 1-point increase) & $0.92(0.84-0.99)$ & 0.03 \\
\hline Onset to treatment delay (per 1-hour increase) & $1.00(0.97-1.03)$ & 0.86 \\
\hline NIHSS score after treatment (per 1-point increase) & $1.13(1.04-1.22)$ & $<0.01$ \\
\hline \multicolumn{3}{|l|}{ Subgroup (intravenous only): $\mathrm{mRS}$ shift at 3 months** $^{*}$} \\
\hline Early statins & $1.17(0.67-2.04)$ & 0.57 \\
\hline Age (per 1-year increase) & $0.95(0.93-0.97)$ & $<0.01$ \\
\hline Male sex & $1.44(0.81-2.54)$ & 0.21 \\
\hline Premorbid $m R S$ score $=0$ & $2.42(1.10-5.31)$ & 0.03 \\
\hline NIHSS score at arrival (per 1-point increase) & $1.01(0.96-1.07)$ & 0.66 \\
\hline Onset to treatment delay (per 1-hour increase) & $0.986(0.968-1.004)$ & 0.13 \\
\hline NIHSS score after treatment (per 1-point increase) & $0.82(0.77-0.87)$ & $<0.01$ \\
\hline \multicolumn{3}{|l|}{ Stroke mechanism } \\
\hline Large artery atherosclerosis & Referent & - \\
\hline Small vessel occlusion & $0.99(0.47-2.09)$ & 0.97 \\
\hline Cardioembolism & $1.76(0.83-3.69)$ & 0.14 \\
\hline Other determined cause & $0.61(0.18-2.02)$ & 0.42 \\
\hline Undetermined cause & $1.07(0.53-2.13)$ & 0.86 \\
\hline Hypertension & $1.58(0.98-2.56)$ & 0.06 \\
\hline Hyperlipidemia & $0.92(0.46-1.84)$ & 0.82 \\
\hline Atrial fibrillation & $0.97(0.48-2.00)$ & 0.94 \\
\hline Regular smoking & $1.16(0.65-2.10)$ & 0.61 \\
\hline Pre-stroke antithrombotics & $0.90(0.52-1.56)$ & 0.71 \\
\hline Pre-stroke statins & $1.03(0.47-2.24)$ & 0.94 \\
\hline Hemoglobin (per $1 \mathrm{~g} / \mathrm{dL}$ increase) & $0.92(0.81-1.05)$ & 0.23 \\
\hline
\end{tabular}


Supplementary Table 8. Continued

\begin{tabular}{|c|c|c|}
\hline Variables & Adjusted OR $(95 \% \mathrm{Cl})$ & $P$ \\
\hline HbA1c (per 1\% increase) & $0.88(0.73-1.04)$ & 0.14 \\
\hline Triglycerides (per 1 mg/dL increase) & $0.999(0.996-1.003)$ & 0.69 \\
\hline LDL cholesterol (per 1 mg/dL increase) & $0.992(0.985-0.999)$ & 0.03 \\
\hline \multicolumn{3}{|l|}{ Subgroup (intravenous only): mRS 0-1 at 3 months $^{+}$} \\
\hline Early statins & $1.20(0.56-2.54)$ & 0.64 \\
\hline Age & $0.53(0.36-0.78)$ & $<0.01$ \\
\hline Male sex & $1.61(0.76-3.41)$ & 0.21 \\
\hline Premorbid mRS score $=0$ & $2.53(0.83-7.72)$ & 0.10 \\
\hline NIHSS score at arrival (per 1-point increase) & $1.05(0.97-1.15)$ & 0.24 \\
\hline Onset to treatment delay (per 1-hour increase) & $0.97(0.94-1.01)$ & 0.13 \\
\hline NIHSS score after treatment (per 1-point increase) & $0.81(0.74-0.89)$ & $<0.01$ \\
\hline \multicolumn{3}{|l|}{ Stroke mechanism } \\
\hline Large artery atherosclerosis & Referent & - \\
\hline Small vessel occlusion & $0.70(0.26-1.89)$ & 0.49 \\
\hline Cardioembolism & $1.84(0.67-5.09)$ & 0.24 \\
\hline Other determined cause & $0.81(0.16-4.15)$ & 0.80 \\
\hline Undetermined cause & $1.22(0.49-3.03)$ & 0.66 \\
\hline Hypertension & $1.45(0.76-2.79)$ & 0.26 \\
\hline Hyperlipidemia & $1.41(0.53-3.75)$ & 0.49 \\
\hline Atrial fibrillation & $0.68(0.26-1.79)$ & 0.43 \\
\hline Regular smoking & $1.25(0.59-2.64)$ & 0.56 \\
\hline Pre-stroke antithrombotics & $0.74(0.35-1.56)$ & 0.43 \\
\hline Pre-stroke statins & $0.86(0.29-2.55)$ & 0.78 \\
\hline Hemoglobin (per $1 \mathrm{~g} / \mathrm{dL}$ increase) & $0.93(0.78-1.10)$ & 0.41 \\
\hline HbA1c (per 1\% increase) & $0.70(0.53-0.93)$ & 0.01 \\
\hline Triglycerides & $0.90(0.66-1.24)$ & 0.52 \\
\hline LDL cholesterol & $0.71(0.50-1.00)$ & 0.05 \\
\hline \multicolumn{3}{|l|}{ Subgroup (intravenous only): symptomatic HT } \\
\hline Early statins & $0.85(0.18-3.87)$ & 0.83 \\
\hline Age (per 1-year increase) & $1.05(0.98-1.12)$ & 0.14 \\
\hline Male sex & $4.02(0.84-19.16)$ & 0.08 \\
\hline Premorbid mRS score $=0$ & $0.53(0.09-3.02)$ & 0.47 \\
\hline NIHSS score at arrival (per 1-point increase) & $0.91(0.79-1.05)$ & 0.18 \\
\hline Onset to treatment delay (per 1-hour increase) & $0.66(0.38-1.16)$ & 0.15 \\
\hline NIHSS score after treatment (per 1-point increase) & $1.20(1.05-1.36)$ & 0.01 \\
\hline \multicolumn{3}{|c|}{ Subgroup (large artery atherosclerosis): mRS shift at 3 months ${ }^{t+,+\neq}$} \\
\hline Early statins & $2.02(1.07-3.82)$ & 0.03 \\
\hline Age (per 1-year increase) & $0.96(0.94-0.99)$ & $<0.01$ \\
\hline Male sex & $1.27(0.66-2.43)$ & 0.48 \\
\hline Premorbid mRS score $=0$ & $1.65(0.70-3.90)$ & 0.25 \\
\hline NIHSS score at arrival (per 1-point increase) & $1.03(0.98-1.08)$ & 0.26 \\
\hline Onset to treatment delay (per 1-hour increase) & $1.01(0.99-1.03)$ & 0.28 \\
\hline \multicolumn{3}{|l|}{ Recanalization treatment } \\
\hline Intravenous thrombolysis alone & Referent & - \\
\hline Endovascular therapy alone & $1.07(0.58-1.99)$ & 0.83 \\
\hline Combined & $0.76(0.39-1.49)$ & 0.43 \\
\hline NIHSS score after treatment (per 1-point increase) & $0.82(0.78-0.87)$ & $<0.01$ \\
\hline Hypertension & $1.53(0.86-2.73)$ & 0.15 \\
\hline Hyperlipidemia & $0.80(0.41-1.55)$ & 0.51 \\
\hline
\end{tabular}


Supplementary Table 8. Continued

\begin{tabular}{|c|c|c|}
\hline Variables & Adjusted OR $(95 \% \mathrm{Cl})$ & $P$ \\
\hline Regular smoking & $1.17(0.64-2.14)$ & 0.61 \\
\hline Pre-stroke antithrombotics & $0.64(0.35-1.17)$ & 0.15 \\
\hline Pre-stroke statins & $0.92(0.43-1.97)$ & 0.84 \\
\hline Hemoglobin (per $1 \mathrm{~g} / \mathrm{dL}$ increase) & $1.03(0.89-1.18)$ & 0.72 \\
\hline HbA1c (per 1\% increase) & $0.87(0.72-1.05)$ & 0.15 \\
\hline Triglycerides (per 1 mg/dL increase) & $1.001(0.997-1.005)$ & 0.61 \\
\hline LDL cholesterol (per 1 mg/dL increase) & $0.994(0.987-1.001)$ & 0.07 \\
\hline \multicolumn{3}{|c|}{ Subgroup (large artery atherosclerosis): mRS 0-1 at 3 month $^{+,+t}$} \\
\hline Early statins & $2.39(0.90-6.40)$ & 0.08 \\
\hline Age & $0.67(0.45-1.02)$ & 0.06 \\
\hline Male sex & $1.56(0.59-4.10)$ & 0.37 \\
\hline Premorbid $m R S$ score $=0$ & $1.43(0.35-5.85)$ & 0.62 \\
\hline NIHSS score at arrival (per 1-point increase) & $1.06(0.97-1.14)$ & 0.19 \\
\hline Onset to treatment delay (per 1-hour increase) & $0.99(0.96-1.02)$ & 0.52 \\
\hline \multicolumn{3}{|l|}{ Recanalization treatment } \\
\hline Intravenous thrombolysis alone & Referent & - \\
\hline Endovascular therapy alone & $1.05(0.44-2.49)$ & 0.91 \\
\hline Combined & $0.38(0.15-0.98)$ & 0.05 \\
\hline NIHSS score after treatment (per 1-point increase) & $0.81(0.74-0.89)$ & $<0.01$ \\
\hline Hypertension & $0.84(0.38-1.87)$ & 0.67 \\
\hline Hyperlipidemia & $1.48(0.54-4.10)$ & 0.45 \\
\hline Regular smoking & $1.38(0.61-3.14)$ & 0.44 \\
\hline Pre-stroke antithrombotics & $0.84(0.36-1.95)$ & 0.68 \\
\hline Pre-stroke statins & $0.90(0.28-2.88)$ & 0.86 \\
\hline Hemoglobin (per $1 \mathrm{~g} / \mathrm{dL}$ increase) & $1.14(0.94-1.38)$ & 0.17 \\
\hline HbA1c (per 1\% increase) & $0.82(0.60-1.11)$ & 0.20 \\
\hline Triglycerides & $0.95(0.68-1.33)$ & 0.75 \\
\hline LDL cholesterol & $0.74(0.52-1.05)$ & 0.09 \\
\hline \multicolumn{3}{|l|}{ Subgroup (large artery atherosclerosis): symptomatic HT } \\
\hline Early statins & $0.19(0.04-0.96)$ & 0.04 \\
\hline Age (per 1-year increase) & $1.33(0.56-3.18)$ & 0.52 \\
\hline Male sex & $0.79(0.17-3.59)$ & 0.76 \\
\hline Premorbid $m R S$ score $=0$ & $1.25(0.15-10.16)$ & 0.83 \\
\hline NIHSS score at arrival (per 1-point increase) & $0.93(0.83-1.05)$ & 0.26 \\
\hline Onset to treatment delay (per 1-hour increase) & $1.01(0.98-1.04)$ & 0.44 \\
\hline \multicolumn{3}{|l|}{ Recanalization treatment } \\
\hline Intravenous thrombolysis alone & Referent & \\
\hline Endovascular therapy alone & $1.01(0.17-5.96)$ & 0.99 \\
\hline Combined & $1.04(0.14-7.60)$ & 0.97 \\
\hline NIHSS score after treatment (per 1-point increase) & $1.11(0.99-1.25)$ & 0.08 \\
\hline \multicolumn{3}{|l|}{ Subgroup (cardioembolism): $\mathrm{mRS}$ shift at 3 months ${ }^{5 \S}$} \\
\hline Early statins & $1.28(0.87-1.88)$ & 0.22 \\
\hline Age (per 1-year increase) & $0.95(0.93-0.97)$ & $<0.01$ \\
\hline Male sex & $0.71(0.46-1.10)$ & 0.13 \\
\hline Premorbid $m R S$ score $=0$ & $1.66(1.01-2.74)$ & 0.05 \\
\hline NIHSS score at arrival (per 1-point increase) & $1.01(0.98-1.05)$ & 0.45 \\
\hline Onset to treatment delay (per 1-hour increase) & $1.00(0.97-1.03)$ & 0.88 \\
\hline \multicolumn{3}{|l|}{ Recanalization treatment } \\
\hline Intravenous thrombolysis alone & Referent & - \\
\hline
\end{tabular}


Supplementary Table 8. Continued

\begin{tabular}{|c|c|c|}
\hline Variables & Adjusted OR (95\% Cl) & $P$ \\
\hline Endovascular therapy alone & $0.76(0.44-1.30)$ & 0.31 \\
\hline Combined & $1.04(0.65-1.66)$ & 0.88 \\
\hline NIHSS score after treatment (per 1-point increase) & $0.82(0.79-0.85)$ & $<0.01$ \\
\hline Hypertension & $1.28(0.84-1.94)$ & 0.25 \\
\hline Hyperlipidemia & $1.04(0.58-1.85)$ & 0.90 \\
\hline Atrial fibrillation & $1.08(0.66-1.79)$ & 0.75 \\
\hline Regular smoking & $1.26(0.80-2.00)$ & 0.32 \\
\hline Pre-stroke antithrombotics & $0.83(0.56-1.23)$ & 0.35 \\
\hline Pre-stroke statins & $1.28(0.70-2.32)$ & 0.42 \\
\hline Hemoglobin (per $1 \mathrm{~g} / \mathrm{dL}$ increase) & $0.99(0.88-1.10)$ & 0.79 \\
\hline HbA1c (per 1\% increase) & $0.82(0.67-1.00)$ & 0.05 \\
\hline Triglycerides (per 1 mg/dL increase) & $1.003(0.999-1.006)$ & 0.13 \\
\hline LDL cholesterol (per 1 mg/dL increase) & $0.993(0.988-0.999)$ & 0.02 \\
\hline \multicolumn{3}{|l|}{ Subgroup (cardioembolism): mRS 0-1 at 3 months $^{+}$} \\
\hline Early statins & $1.32(0.74-2.37)$ & 0.35 \\
\hline Age & $0.66(0.47-0.91)$ & 0.01 \\
\hline Male sex & $0.62(0.32-1.20)$ & 0.16 \\
\hline Premorbid $\mathrm{mRS}$ score $=0$ & $1.76(0.72-4.34)$ & 0.22 \\
\hline NIHSS score at arrival (per 1-point increase) & $0.99(0.94-1.04)$ & 0.69 \\
\hline Onset to treatment delay (per 1-hour increase) & $0.99(0.95-1.03)$ & 0.52 \\
\hline \multicolumn{3}{|l|}{ Recanalization treatment } \\
\hline Intravenous thrombolysis alone & Referent & - \\
\hline Endovascular therapy alone & $0.60(0.27-1.30)$ & 0.19 \\
\hline Combined & $1.14(0.58-2.21)$ & 0.71 \\
\hline NIHSS score after treatment (per 1-point increase) & $0.82(0.77-0.87)$ & $<0.01$ \\
\hline Hypertension & $1.22(0.64-2.31)$ & 0.55 \\
\hline Hyperlipidemia & $1.20(0.48-2.97)$ & 0.70 \\
\hline Atrial fibrillation & $1.19(0.57-2.45)$ & 0.64 \\
\hline Regular smoking & $1.81(0.92-3.56)$ & 0.08 \\
\hline Pre-stroke antithrombotics & $0.70(0.38-1.27)$ & 0.24 \\
\hline Pre-stroke statins & $1.06(0.41-2.76)$ & 0.90 \\
\hline Hemoglobin (per $1 \mathrm{~g} / \mathrm{dL}$ increase) & $0.95(0.80-1.12)$ & 0.54 \\
\hline HbA1c (per 1\% increase) & $0.97(0.73-1.30)$ & 0.86 \\
\hline Triglycerides & $1.23(0.93-1.64)$ & 0.15 \\
\hline LDL cholesterol & $0.68(0.50-0.91)$ & 0.01 \\
\hline \multicolumn{3}{|l|}{ Subgroup (cardioembolism): symptomatic HT } \\
\hline Early statins & $0.60(0.26-1.38)$ & 0.23 \\
\hline Age (per 1-year increase) & $0.98(0.94-1.01)$ & 0.21 \\
\hline Male sex & $1.09(0.49-2.44)$ & 0.83 \\
\hline Premorbid $\mathrm{mRS}$ score $=0$ & $1.19(0.40-3.47)$ & 0.76 \\
\hline NIHSS score at arrival (per 1-point increase) & $0.91(0.84-0.98)$ & 0.02 \\
\hline Onset to treatment delay (per 1-hour increase) & $0.97(0.89-1.06)$ & 0.53 \\
\hline \multicolumn{3}{|l|}{ Recanalization treatment } \\
\hline Intravenous thrombolysis alone & Referent & - \\
\hline Endovascular therapy alone & $1.69(0.49-5.82)$ & 0.41 \\
\hline Combined & $1.11(0.38-3.21)$ & 0.85 \\
\hline NIHSS score after treatment (per 1-point increase) & $1.15(1.07-1.23)$ & $<0.01$ \\
\hline \multicolumn{3}{|c|}{ Subgroup (other stroke mechanisms): mRS shift at 3 months ${ }^{11 ! I}$} \\
\hline Early statins & $1.89(1.02-3.53)$ & 0.04 \\
\hline
\end{tabular}


Supplementary Table 8. Continued

\begin{tabular}{|c|c|c|}
\hline Variables & Adjusted OR $(95 \% \mathrm{Cl})$ & $P$ \\
\hline Age (per 1-year increase) & $0.97(0.95-0.99)$ & 0.01 \\
\hline Male sex & $0.46(0.23-0.94)$ & 0.03 \\
\hline Premorbid $m R S$ score $=0$ & $1.84(0.80-4.23)$ & 0.15 \\
\hline NIHSS score at arrival (per 1-point increase) & $1.06(0.997-1.12)$ & 0.06 \\
\hline Onset to treatment delay (per 1-hour increase) & $0.99(0.97-1.01)$ & 0.19 \\
\hline \multicolumn{3}{|l|}{ Recanalization treatment } \\
\hline Intravenous thrombolysis alone & Referent & - \\
\hline Endovascular therapy alone & $0.82(0.42-1.58)$ & 0.55 \\
\hline Combined & $0.99(0.50-1.95)$ & 0.98 \\
\hline NIHSS score after treatment (per 1-point increase) & $0.80(0.75-0.85)$ & $<0.01$ \\
\hline Hypertension & $1.19(0.67-2.12)$ & 0.55 \\
\hline Hyperlipidemia & $0.70(0.28-1.77)$ & 0.45 \\
\hline Atrial fibrillation & $0.82(0.34-1.99)$ & 0.66 \\
\hline Regular smoking & $2.19(1.10-4.36)$ & 0.03 \\
\hline Pre-stroke antithrombotics & $1.33(0.71-2.49)$ & 0.37 \\
\hline Pre-stroke statins & $2.36(0.79-7.07)$ & 0.12 \\
\hline Hemoglobin (per $1 \mathrm{~g} / \mathrm{dL}$ increase) & $1.25(1.08-1.44)$ & $<0.01$ \\
\hline $\mathrm{HbA} 1 \mathrm{c}$ (per 1\% increase) & $0.83(0.66-1.06)$ & 0.14 \\
\hline Triglycerides (per 1 mg/dL increase) & $1.000(0.996-1.003)$ & 0.84 \\
\hline LDL cholesterol (per 1 mg/dL increase) & $0.999(0.991-1.006)$ & 0.73 \\
\hline \multicolumn{3}{|c|}{ Subgroup (other stroke mechanisms): mRS 0-1 at 3 months $^{+}$} \\
\hline Early statins & $1.93(0.78-4.79)$ & 0.15 \\
\hline Age & $0.59(0.39-0.89)$ & 0.01 \\
\hline Male sex & $0.45(0.15-1.29)$ & 0.14 \\
\hline Premorbid mRS score $=0$ & $2.07(0.60-7.14)$ & 0.25 \\
\hline NIHSS score at arrival (per 1-point increase) & $1.05(0.97-1.14)$ & 0.23 \\
\hline Onset to treatment delay (per 1-hour increase) & $0.98(0.95-1.01)$ & 0.29 \\
\hline \multicolumn{3}{|l|}{ Recanalization treatment } \\
\hline Intravenous thrombolysis alone & Referent & - \\
\hline Endovascular therapy alone & $0.50(0.19-1.31)$ & 0.16 \\
\hline Combined & $1.14(0.43-3.03)$ & 0.79 \\
\hline NIHSS score after treatment (per 1-point increase) & $0.78(0.70-0.86)$ & $<0.01$ \\
\hline Hypertension & $1.57(0.69-3.54)$ & 0.28 \\
\hline Hyperlipidemia & $1.66(0.48-5.80)$ & 0.43 \\
\hline Atrial fibrillation & $1.20(0.32-4.49)$ & 0.79 \\
\hline Regular smoking & $4.14(1.47-11.64)$ & 0.01 \\
\hline Pre-stroke antithrombotics & $0.92(0.36-2.35)$ & 0.87 \\
\hline Pre-stroke statins & $1.47(0.33-6.56)$ & 0.62 \\
\hline Hemoglobin (per $1 \mathrm{~g} / \mathrm{dL}$ increase) & $1.03(0.84-1.27)$ & 0.74 \\
\hline HbA1c (per 1\% increase) & $0.69(0.47-1.02)$ & 0.06 \\
\hline Triglycerides & $0.98(0.69-1.39)$ & 0.91 \\
\hline LDL cholesterol & $0.96(0.65-1.43)$ & 0.86 \\
\hline \multicolumn{3}{|l|}{ Subgroup (other stroke mechanisms): symptomatic HT } \\
\hline Early statins & $0.49(0.12-1.99)$ & 0.32 \\
\hline Age (per 1-year increase) & $1.02(0.97-1.08)$ & 0.43 \\
\hline Male sex & $3.19(0.68-14.93)$ & 0.14 \\
\hline Premorbid mRS score $=0$ & $0.94(0.15-5.86)$ & 0.95 \\
\hline NIHSS score at arrival (per 1-point increase) & $0.94(0.83-1.07)$ & 0.36 \\
\hline Onset to treatment delay (per 1-hour increase) & $0.98(0.89-1.08)$ & 0.72 \\
\hline
\end{tabular}


Supplementary Table 8. Continued

\begin{tabular}{|c|c|c|}
\hline Variables & Adjusted OR (95\% Cl) & $P$ \\
\hline \multicolumn{3}{|l|}{ Recanalization treatment } \\
\hline Intravenous thrombolysis alone & Referent & - \\
\hline Endovascular therapy alone & $1.00(0.16-6.35)$ & 1.00 \\
\hline Combined & $1.34(0.24-7.59)$ & 0.74 \\
\hline NIHSS score after treatment (per 1-point increase) & $1.13(1.01-1.27)$ & 0.03 \\
\hline \multicolumn{3}{|l|}{ Subgroup (pre-stroke statins: yes): mRS shift at 3 months" } \\
\hline Early statins & $1.58(0.84-2.99)$ & 0.16 \\
\hline Age (per 1-year increase) & $0.96(0.92-0.99)$ & 0.01 \\
\hline Male sex & $0.91(0.45-1.84)$ & 0.78 \\
\hline Premorbid mRS score $=0$ & $2.85(1.35-5.99)$ & 0.01 \\
\hline NIHSS score at arrival (per 1-point increase) & $1.01(0.95-1.07)$ & 0.82 \\
\hline Onset to treatment delay (per 1-hour increase) & $1.00(0.98-1.03)$ & 0.77 \\
\hline \multicolumn{3}{|l|}{ Recanalization treatment } \\
\hline Intravenous thrombolysis alone & Referent & - \\
\hline Endovascular therapy alone & $1.37(0.65-2.91)$ & 0.40 \\
\hline Combined & $1.30(0.64-2.66)$ & 0.47 \\
\hline NIHSS score after treatment (per 1-point increase) & $0.80(0.75-0.85)$ & $<0.01$ \\
\hline \multicolumn{3}{|l|}{ Stroke mechanism } \\
\hline Large artery atherosclerosis & Referent & \\
\hline Small vessel occlusion & $4.00(0.54-29.51)$ & 0.17 \\
\hline Cardioembolism & $2.32(0.85-6.33)$ & 0.10 \\
\hline Other determined cause & $0.41(0.06-2.82)$ & 0.36 \\
\hline Undetermined cause & $1.89(0.78-4.61)$ & 0.16 \\
\hline Hypertension & $1.54(0.73-3.23)$ & 0.26 \\
\hline Hyperlipidemia & $0.71(0.27-1.87)$ & 0.49 \\
\hline Atrial fibrillation & $0.92(0.37-2.26)$ & 0.85 \\
\hline Regular smoking & $0.90(0.45-1.83)$ & 0.78 \\
\hline Pre-stroke antithrombotics & $1.61(0.84-3.06)$ & 0.15 \\
\hline Hemoglobin (per $1 \mathrm{~g} / \mathrm{dL}$ increase) & $1.15(0.97-1.38)$ & 0.11 \\
\hline HbA1c (per 1\% increase) & $1.21(0.91-1.60)$ & 0.19 \\
\hline Triglycerides (per 1 mg/dL increase) & $1.00(0.99-1.01)$ & 0.81 \\
\hline LDL cholesterol (per 1 mg/dL increase) & $0.993(0.984-1.001)$ & 0.09 \\
\hline \multicolumn{3}{|l|}{ Subgroup (pre-stroke statins: yes): mRS 0-1 at 3 months $^{+}$} \\
\hline Early statins & $2.63(0.94-7.38)$ & 0.07 \\
\hline Age & $0.60(0.36-1.00)$ & 0.0499 \\
\hline Male sex & $0.62(0.21-1.82)$ & 0.38 \\
\hline Premorbid mRS score $=0$ & $2.34(0.68-8.09)$ & 0.18 \\
\hline NIHSS score at arrival (per 1-point increase) & $0.98(0.91-1.07)$ & 0.67 \\
\hline Onset to treatment delay (per 1-hour increase) & $0.99(0.96-1.03)$ & 0.61 \\
\hline \multicolumn{3}{|l|}{ Recanalization treatment } \\
\hline Intravenous thrombolysis alone & Referent & - \\
\hline Endovascular therapy alone & $1.71(0.58-5.06)$ & 0.33 \\
\hline Combined & $1.31(0.47-3.68)$ & 0.60 \\
\hline NIHSS score after treatment (per 1-point increase) & $0.82(0.75-0.90)$ & $<0.01$ \\
\hline \multicolumn{3}{|l|}{ Stroke mechanism } \\
\hline Large artery atherosclerosis & Referent & - \\
\hline Small vessel occlusion & $6.66(0.43-103.28)$ & 0.17 \\
\hline Cardioembolism & $1.38(0.32-5.97)$ & 0.67 \\
\hline Other determined cause & $0.62(0.04-10.76)$ & 0.74 \\
\hline
\end{tabular}


Supplementary Table 8. Continued

\begin{tabular}{|c|c|c|}
\hline Variables & Adjusted OR (95\% Cl) & $P$ \\
\hline Undetermined cause & $0.92(0.25-3.36)$ & 0.90 \\
\hline Hypertension & $1.84(0.57-5.89)$ & 0.30 \\
\hline Hyperlipidemia & $1.71(0.37-7.95)$ & 0.49 \\
\hline Atrial fibrillation & $1.18(0.31-4.55)$ & 0.81 \\
\hline Regular smoking & $1.14(0.41-3.12)$ & 0.80 \\
\hline Pre-stroke antithrombotics & $2.04(0.74-5.60)$ & 0.17 \\
\hline Hemoglobin (per $1 \mathrm{~g} / \mathrm{dL}$ increase) & $1.09(0.84-1.43)$ & 0.50 \\
\hline HbA1c (per 1\% increase) & $1.27(0.85-1.88)$ & 0.24 \\
\hline Triglycerides & $1.06(0.69-1.63)$ & 0.79 \\
\hline LDL cholesterol & $0.60(0.39-0.94)$ & 0.02 \\
\hline \multicolumn{3}{|l|}{ Subgroup (pre-stroke statins: yes): symptomatic HT } \\
\hline Early statins & $0.16(0.04-0.60)$ & 0.01 \\
\hline Age (per 1-year increase) & $0.99(0.92-1.07)$ & 0.87 \\
\hline Male sex & $2.31(0.57-9.39)$ & 0.24 \\
\hline Premorbid $m R S$ score $=0$ & $0.73(0.16-3.33)$ & 0.68 \\
\hline NIHSS score at arrival (per 1-point increase) & $0.99(0.86-1.14)$ & 0.92 \\
\hline Onset to treatment delay (per 1-hour increase) & $1.04(0.996-1.08)$ & 0.08 \\
\hline \multicolumn{3}{|l|}{ Recanalization treatment } \\
\hline Intravenous thrombolysis alone & Referent & - \\
\hline Endovascular therapy alone & $0.17(0.02-1.79)$ & 0.14 \\
\hline Combined & $1.30(0.28-6.09)$ & 0.74 \\
\hline NIHSS score after treatment (per 1-point increase) & $1.10(0.97-1.24)$ & 0.13 \\
\hline \multicolumn{3}{|l|}{ Subgroup (pre-stroke statins: no): mRS shift at 3 months ${ }^{* * *}$} \\
\hline Early statins & $1.43(1.03-1.98)$ & 0.03 \\
\hline Age (per 1-year increase) & $0.96(0.95-0.97)$ & $<0.01$ \\
\hline Male sex & $0.73(0.51-1.04)$ & 0.08 \\
\hline Premorbid $m R S$ score $=0$ & $1.39(0.88-2.18)$ & 0.16 \\
\hline NIHSS score at arrival (per 1-point increase) & $1.03(1.004-1.06)$ & 0.03 \\
\hline Onset to treatment delay (per 1-hour increase) & $1.00(0.99-1.01)$ & 0.90 \\
\hline \multicolumn{3}{|l|}{ Recanalization treatment } \\
\hline Intravenous thrombolysis alone & Referent & \\
\hline Endovascular therapy alone & $0.77(0.52-1.13)$ & 0.18 \\
\hline Combined & $0.98(0.67-1.43)$ & 0.91 \\
\hline NIHSS score after treatment (per 1-point increase) & $0.82(0.79-0.84)$ & $<0.01$ \\
\hline \multicolumn{3}{|l|}{ Stroke mechanism } \\
\hline Large artery atherosclerosis & Referent & \\
\hline Small vessel occlusion & $0.70(0.33-1.46)$ & 0.34 \\
\hline Cardioembolism & $1.45(0.87-2.43)$ & 0.16 \\
\hline Other determined cause & $0.25(0.12-0.53)$ & $<0.01$ \\
\hline Undetermined cause & $0.84(0.54-1.31)$ & 0.44 \\
\hline Hypertension & $1.28(0.93-1.75)$ & 0.13 \\
\hline Hyperlipidemia & $0.70(0.42-1.15)$ & 0.16 \\
\hline Atrial fibrillation & $0.78(0.48-1.26)$ & 0.31 \\
\hline Regular smoking & $1.68(1.17-2.40)$ & $<0.01$ \\
\hline Pre-stroke antithrombotics & $0.81(0.59-1.12)$ & 0.20 \\
\hline Hemoglobin (per $1 \mathrm{~g} / \mathrm{dL}$ increase) & $1.04(0.96-1.13)$ & 0.35 \\
\hline HbA1c (per 1\% increase) & $0.77(0.67-0.89)$ & $<0.01$ \\
\hline Triglycerides (per 1 mg/dL increase) & $1.001(0.999-1.003)$ & 0.55 \\
\hline LDL cholesterol (per 1 mg/dL increase) & $0.998(0.994-1.003)$ & 0.46 \\
\hline
\end{tabular}


Supplementary Table 8. Continued

\begin{tabular}{|c|c|c|}
\hline Variables & Adjusted OR (95\% Cl) & $P$ \\
\hline \multicolumn{3}{|l|}{ Subgroup (pre-stroke statins: no): mRS 0-1 at 3 months $^{+}$} \\
\hline Early statins & $1.36(0.84-2.20)$ & 0.22 \\
\hline Age & $0.66(0.51-0.84)$ & $<0.01$ \\
\hline Male sex & $0.77(0.45-1.32)$ & 0.35 \\
\hline Premorbid mRS score $=0$ & $1.81(0.82-4.00)$ & 0.14 \\
\hline NIHSS score at arrival (per 1-point increase) & $1.02(0.98-1.07)$ & 0.31 \\
\hline Onset to treatment delay (per 1-hour increase) & $0.98(0.96-1.01)$ & 0.16 \\
\hline \multicolumn{3}{|l|}{ Recanalization treatment } \\
\hline Intravenous thrombolysis alone & Referent & - \\
\hline Endovascular therapy alone & $0.53(0.31-0.93)$ & 0.03 \\
\hline Combined & $0.80(0.47-1.35)$ & 0.40 \\
\hline NIHSS score after treatment (per 1-point increase) & $0.81(0.77-0.85)$ & $<0.01$ \\
\hline \multicolumn{3}{|l|}{ Stroke mechanism } \\
\hline Large artery atherosclerosis & Referent & - \\
\hline Small vessel occlusion & $0.38(0.14-1.02)$ & 0.05 \\
\hline Cardioembolism & $1.61(0.75-3.47)$ & 0.22 \\
\hline Other determined cause & $0.67(0.24-1.86)$ & 0.44 \\
\hline Undetermined cause & $0.92(0.49-1.72)$ & 0.78 \\
\hline Hypertension & $1.06(0.68-1.66)$ & 0.79 \\
\hline Hyperlipidemia & $0.98(0.48-2.03)$ & 0.96 \\
\hline Atrial fibrillation & $0.95(0.46-1.95)$ & 0.89 \\
\hline Regular smoking & $2.44(1.46-4.10)$ & $<0.01$ \\
\hline Pre-stroke antithrombotics & $0.68(0.42-1.10)$ & 0.12 \\
\hline Hemoglobin (per $1 \mathrm{~g} / \mathrm{dL}$ increase) & $1.03(0.92-1.16)$ & 0.61 \\
\hline HbA1c (per 1\% increase) & $0.75(0.60-0.93)$ & 0.01 \\
\hline Triglycerides & $0.98(0.80-1.21)$ & 0.88 \\
\hline LDL cholesterol & $0.93(0.75-1.16)$ & 0.54 \\
\hline \multicolumn{3}{|l|}{ Subgroup (pre-stroke statins: no): symptomatic HT } \\
\hline Early statins & $0.16(0.04-0.60)$ & 0.01 \\
\hline Age (per 1-year increase) & $0.99(0.92-1.07)$ & 0.87 \\
\hline Male sex & $2.31(0.57-9.39)$ & 0.24 \\
\hline Premorbid mRS score $=0$ & $0.73(0.16-3.33)$ & 0.68 \\
\hline NIHSS score at arrival (per 1-point increase) & $0.99(0.86-1.14)$ & 0.92 \\
\hline Onset to treatment delay (per 1-hour increase) & $1.04(0.996-1.08)$ & 0.08 \\
\hline \multicolumn{3}{|l|}{ Recanalization treatment } \\
\hline Intravenous thrombolysis alone & Referent & - \\
\hline Endovascular therapy alone & $0.17(0.02-1.79)$ & 0.14 \\
\hline Combined & $1.30(0.28-6.09)$ & 0.74 \\
\hline NIHSS score after treatment (per 1-point increase) & $1.10(0.97-1.24)$ & 0.13 \\
\hline
\end{tabular}

$\mathrm{OR}$, odds ratio; Cl, Contidence interval; mRS, modified Rankin Scale; NIHSS, National Institute of Health Stroke Scale; LDL, low-density lipoprotein; IV, intravenous; $\mathrm{HT}$, hemorrhage transformation.

${ }^{*}$ Likelihood ratio test of non-proportional odds for early statins: $P=0.16 ;{ }^{\dagger}$ Age, triglycerides, and LDL cholesterol were standardized for the mixed model; ${ }^{*}$ Likelihood ratio test of non-proportional odds for early statins: $P=0.76$; ${ }^{5}$ Likelihood ratio test of non-proportional odds for early statins: $P=0.55$; "Likelihood ratio test of non-proportional odds for early statins: $P=0.58$; 'Likelihood ratio test of non-proportional odds for early statins: $P=0.67$; **ikelihood ratio test of nonproportional odds for early statins: $P=0.80 ;{ }^{+\dagger}$ Atrial fibrillation was excluded from the model due to its singularity; ${ }^{* \dagger}$ Likelihood ratio test of non-proportional odds for early statins: $P=0.95$; $\S \S$ Likelihood ratio test of non-proportional odds for early statins: $P=0.77$; "IILikelihood ratio test of non-proportional odds for early statins: $P=0.33$; "Likelihood ratio test of non-proportional odds for early statins: $P=0.24$; ${ }^{* *}$ Likelihood ratio test of non-proportional odds for early statins: $P=0.04$. 